\title{
A SPATIO-TEMPORAL MODELING FRAMEWORK FOR WEATHER RADAR IMAGE DATA IN TROPICAL SOUTHEAST ASIA
}

\author{
By XiaO LiU, ViKnESWARAN GOPAL AND JAYANT KALAGNANAM \\ University of Arkansas, National University of Singapore, and IBM Thomas J. \\ Watson Research Center
}

\begin{abstract}
Tropical storms are known to be highly chaotic and extremely difficult to predict. In tropical countries such as Singapore, the official lead time for the warnings of heavy storms is usually between 15 and 45 minutes because weather systems develop quickly and are of very short lifespan. A single thunderstorm cell, for example, typically lives for less than an hour. Weather radar echoes, correlated in both space and time, provide a rich source of information for short-term precipitation nowcasting. Based on a large dataset of 276 tropical storms events, this paper investigates a spatio-temporal modeling approach for two-dimensional radar reflectivity (echo) fields. Under a Lagrangian integration scheme, we model the radar reflectivity field by a spatiotemporal conditional autoregressive process with two components. The first component is the dynamic velocity field which determines the motion of the storm, and the second component governs the growth or decay of the returned radar echoes. The proposed method is demonstrated and compared with existing methods using real radar image data collected from a number of 276 tropical storm events from 2010 to 2011 in Singapore. The numerical comparison results show the advantage of the proposed method, in terms of the meansquared-error, in modeling small-scale localized convective weather systems based on the 77 inter-monsoon season thunderstorm events.
\end{abstract}

\section{Introduction.}

1.1. Background. The intensity of precipitation (rain, snow, and sleet) can be estimated from the amount of transmitted power returned to the weather radar receiver. Since the discovery of such a phenomenon during World War II, various methods have been developed to locate, track, and predict precipitation, with the fundamental idea in common being the spatio-temporal extrapolation/advection of radar reflectivity (echo) field. Nowadays, these methods are collectively known as the radar-based Quantitative Precipitation Forecasts (QPF) in the meteorological community [Wilson et al. (1998), RMI (2008)].

The motion of a weather system, characterized by a velocity vector field, is not directly observed and needs to be estimated from a sequence of images for the spatio-temporal extrapolation of radar reflectivity field. In the past two

Received August 2016; revised May 2017.

Key words and phrases. Spatial-temporal modeling, precipitation forecast, space-time conditional autoregressive model, radar image analysis. 
decades, four types of methods prevail in practice: (1) gradient-based (intensityinvariant) method, (2) pattern-based (shape-invariant) methods, (3) cell tracking, and (4) spectral approach. Gradient-based methods, proposed by Horn and Schunck (1981), assume that reflectivity level is time-invariant and construct the velocity vector field based on the first-order variation of reflectivity. Although often known as the Optical Flow (OF) in computer science (as it was proposed in studying the two-dimensional velocities of the brightness patterns of image sequences), gradient-based methods are referred to as the method of Lagrangian persistence by meteorologists, and are now the building block of many storm-tracking algorithms [Bowler, Pierce and Seed (2004)]. Pattern-based methods, on the other hand, are based on the concept of area tracking. Tracking areas (i.e., radar image pixel arrays) are defined around all pixel grid points, and corresponding areas are searched in the next radar image by maximizing the cross-correlation between areas. Then the velocity field can be constructed given the spatial lags between areas and the time lag between two radar scans. Unlike gradient-based methods, pattern-based methods assume that the shape of reflectivity patterns within defined areas do not change over short time intervals. In the literature, pattern-based methods were first introduced by Leese, Novak and Clark (1971) to identify cloud motion from satellite images, and has later been successfully used for the nowcasting of precipitation with radar over a complex orography [Li, Schmid and Joss (1995), Rinehart and Garvey (1978), Li and Lai (2004)]. Cell tracking methods detect storm cells as contiguous regions of enhanced reflectivity exceeding a certain threshold in both reflectivity and volume, and concentrate on the development and displacement of these cells. For example, the TITAN algorithm (Thunderstorm Identification Tracking Analysis and Nowcasting) is one of the most widely used algorithms under this category [Dixon and Wiener (1993), Han et al. (2009)]. Spectral methods are based on the idea that rain fields commonly exhibit both spatial and dynamic scaling properties, and the smallest scales in radar images usually have shorter lifetimes and are the least predictable [Seed (2003)]. In Appendix B, we provide a brief summary of the operational QPF systems which are developed and deployed by different countries. As different strategies have their own advantages when they are applied to a certain type of weather systems for different purposes, ensemble methods are widely adopted by these systems in practice [Seed, Pierce and Norman (2013)]. Readers may also refer to Gelpke and Künsch (2001) for a comprehensive review of the statistical methods for motion estimation.

The estimation of the velocity vector field serves as a preliminary but necessary step for the spatio-temporal modeling framework to be described in this paper. Our objective is not to improve any existing method for estimating the velocity vector field, but to investigate a general statistical modeling framework that allows us to integrate an existing method with a spatio-temporal model that captures some special features of the storm systems in Southeast Asia, which are to be explained in the next subsection. 
Given the observed or extrapolated radar reflectivity, precipitation intensity can be estimated through some reflectivity-rainfall relationships [Testik and Gebremichael (2013)]; for example, the Marshall-Palmer relationship between radar reflectivity and the distribution of the size of rain drops [Marshall and Palmer (1948)]. Compared with pure physical relationships, statistical models are often more effective in capturing the uncertainty and dynamics associated with the radarrainfall relationship. Brown et al. (2001) developed a high dimensional multivariate state space time series model for space-time calibration of radar-rainfall data. Fuentes, Reich and Lee (2008) introduced a framework, based on the spatial logistic regression, that combines radar reflectivity and gage rainfall data by expressing the different sources of rainfall information in terms of an underlying unobservable spatial temporal process with the true rainfall values. $\mathrm{Xu}$, Wikle and Fox (2005) proposed a kernel-based radar reflectivity nowcasting approach that efficiently parameterizes spatio-temporal dynamic models in terms of integrodifference equations within a hierarchical framework. The kernel-based method has certain advantages when incorporating the underlying physics into the rainfall process. A good example is presented by Sigrist, Künsch and Stahel (2012) in which the authors presented a hierarchical Bayesian model for short-term predictions of rainfall, based on temporal autoregressive convolution with spatially colored and temporally white innovations.

1.2. The modeling of weather radar data in tropical Southeast Asia. The characteristics of storm systems at different geo-locations can be fundamentally different. This paper focuses on the spatio-temporal modeling of weather radar reflectivity data recorded in Southeast Asia by Singapore Meteorological Service. Given the unique behaviors and physics behind the weather systems in this region, special considerations are needed to address the following challenges.

The most important type of storm system in Singapore is known as the smallscale localized convective weather system. As a tropical country, Singapore is located in an active area known as the Inter-tropical Convergence Zone, where weather systems are highly chaotic, and thus difficult to predict. This is a special area where trade winds from both hemispheres meet. Hence, the winds near the equator are generally light but highly variable. More importantly, the strong solar heating of land areas in tropical areas causes a phenomenon known as the convective heating. As a result, the land areas become heated more than its surroundings, leading to significant evaporation that creates the small-scale localized convective weather system. For such a weather system, heavy thunderstorms can develop, grow, and dissipate very suddenly in a random manner. The lifetime of convective thunderstorm cells can be as short as tens of minutes, posing a tremendous challenge to the modeling of radar reflectivity data. Because of this, the official lead time for the warnings of heavy storms in Singapore is between 15 and 45 minutes, and is among the shortest in the world [NEA (2017)]. In 1963, the American meteorologist, Edward Lorenz, had already pinned down the chaotic nature of 
atmospheric convection for weather forecasting. The exact physics behind the convective processes which lead to the formation of such thunderstorms is not yet fully understood. In general, the strength of radar reflectivity can grow or decay during the collision-coalescence process due to vertical and horizontal winds, breakup, and evaporation. In tropical areas considered in this paper, the growth and decay of reflectivity become more prominent due to the presence of many small-scale convective storm cells embedded in the storm system.

Hence, it is necessary to consider the small-scale rapid growth and decay in modeling the radar reflectivity data in Southeast Asia. Browning et al. (1982) showed that errors in the linear extrapolation of the radar echo field assuming persistent reflectivity level are mainly due to the growth and decay of reflectivity. But, the prediction of the growth and decay is not trivial and most of the early efforts have not been very successful due to the nature of the problem [Wolfson et al. (1999)]. In fact, many existing operational QPF systems in the world do not take into account the rapid small-scale growth and decay, such as the GANDOLF system in UK [Bowler, Pierce and Seed (2004)] and the SWIRLS system developed in Hong Kong [Li and Lai (2004)]. One operational system that considers the growth and decay of reflectivity is the McGill Algorithm for Precipitation Nowcasting by Lagrangian Extrapolation (MAPLE) [Radhakrishna, Zawadzki and Fabry (2012), Germann and Zawadzki (2002)]. It is pointed out by the authors that the growth and decay of precipitating systems can be estimated by the mismatch between two radar scans under the Lagrangian integration scheme. However, the authors assume that the growth and decay are persistent over time, and a statistical model that exploits both the spatial and temporal correlation of the reflectivity data is not available.

In addition, it is worth noting that the small-scale localized convective weather system also makes the Numerical Weather Prediction (NWP) models ineffective in predicting the exact location and intensity of individual thunderstorms. In particular, for a small country or area like Singapore, NWP models are not able to capture small-scale features including the growth and decay, as the resolutions of a typical NWP simulation model ranges in the tens to hundreds of kilometers.

Based on the discussions above, the stochastic modeling of weather radar data, using statistical techniques, is not only appealing but also necessary in capturing the dynamics of the tropical storm systems considered in this paper.

1.3. Overview. This paper explores a spatio-temporal modeling framework for weather radar reflectivity data. The proposed framework aims to integrate: (1) the existing methods for estimating the velocity vector field of a weather system, and (2) a statistical spatio-temporal model that captures the small-scale growth and decay of radar reflectivity.

We consider a classic forced-advection problem under the Lagrangian frame of reference, where an observer watches the world evolve around her as if she traveled with the radar image pixel arrays within a velocity field. Based on the discrete 
approximation to the forced-advection problem, we adopt the idea as suggested by the Canadian MAPLE system [Germann and Zawadzki (2002), Radhakrishna, Zawadzki and Fabry (2012)], and assess the growth and decay of reflectivity by the mismatch between two radar images given the constructed velocity vector field. The reflectivity growth and decay are then modeled by extending the Spatial Temporal Conditional Autoregressive (STCAR) model described in Mariella and Tarantino (2010). In the literature, the Conditional Regressive Model (CAR) model, or Gauss-Markov model, have been extensively used for modeling geographical area data when a spatial phenomenon at a location is affected by its neighboring areas [Besag and Kooperberg (1995), Carlin and Banerjee (2003), Cressie (1993), Stern and Cressie (2000), Banerjee, Carlin and Gelfand (2015)]. The spatial structure implied by the CAR model has also been investigated by Wall (2004).

As shown in Section 2, a radar image pixel array consists of a number of image pixels and can be naturally modeled as a spatial area. Because the reflectivity at different spatial locations are recorded in a sequence of equally spaced time intervals, the STCAR model (which is essentially an autoregressive model for a temporal sequence of CAR) takes into account both the spatial dependence of the reflectivity growth among neighboring pixel arrays and the temporal dependence of the reflectivity growth on the same pixel array. Unlike many other conventional multivariate geographical area data models, the spatial locations (i.e., the coordinates) of pixel arrays change over time due to the motion of weather systems. Hence, neither the distance nor the spatial relationship between two pixel arrays is time-invariant, leading to a more dynamic and complicated structure of spatial dependence. On the other hand, it is also desirable to keep the model simple and computationally efficient. Note that, an STCAR model can be directly specified by choosing the joint distribution of a sequence of Markov random fields via conditional and marginal distributions. Based on the recent findings of Radhakrishna, Zawadzki and Fabry (2012), the growth and decay of precipitation intensity over short time intervals are usually well approximated by Gaussian. This finding, which is also verified in our application example presented in Section 4, allows us to conveniently specify the conditional and marginal distributions for the STCAR model. Furthermore, compared to other multivariate areal data model, such as the Generalized Multivariate Conditional Auto-egressive (GMCAR) model [Jin, Carlin and Banerjee (2005)], the STCAR model provides a simple yet efficient way to capture the spatial association at a particular time point by a single parameter, leading to a significant reduction in the computational time. Note that, as a new weather radar image typically becomes available every 5 minutes, the model needs to be constructed or updated in a few minutes including the time consumed by data processing and transfer.

The paper is organized as follows. Section 2 describes the radar data as well as the basic settings of a radar image. The general modeling framework and details are provided in Section 3. In Section 4, an application example are presented using 
real radar data. A comparison study is presented in Section 5 based on a number of 276 storm events. Section 6 is the conclusion of the paper and also highlights some future research directions.

2. Motivating example and basic settings. In this section, we provide a motivating example and describe the basic settings of a weather radar image.

2.1. Singapore floods. As a tropical island country located $137 \mathrm{~km}$ north of the equator, Singapore has a tropical rainforest climate with abundant rainfall. Based on a 145-year survey from the year 1869 to 2013, the average yearly rainfall of Singapore is approximately $2344 \mathrm{~mm}$, and the average raining days of a year is 178 days [NEA (2014)]. The yearly rainfall of Singapore is respectively 4 and 2.5 times higher than that of London and Seattle.

The 2010-2013 Singapore floods refer to the series of flash floods that hit various parts of the city state Singapore since 2010. The floods came about due to the higher-than-average rainfall that aggregated over a short period of time. Detailed descriptions and a complete list of flooding incidents are available from Wikipedia under "2010-2013 Singapore floods". Consider, for example, a heavy rain event on the early morning of 25 June 2010. A torrential downpour early that morning triggered flash floods across Singapore. The flood also caused morning rush hour traffic to come to a virtual standstill on all major expressways. It was reported that the heavy downpour on that Friday morning was equivalent to about $60 \%$ of the average monthly rainfall in June. Social media and micro-blogging sites such as Facebook and Twitter were awash with flood photos and users exchanging pictures. Figure 1 shows 7 consecutive weather radar scans of reflectivity taken from 8 AM to 8:30 AM, 25 June 2010. A tropical storm, moving from the west to the east, is clearly identified by the high-reflectivity area (the orange area) in the figures. The last plot in Figure 1 shows the map of the spatial area covered by a single radar scan. Note that, Singapore is the small island (the shaded area) at the center of the image, and the $x$ - and $y$-coordinates are based on the SVY21 projection which has a one-to-one correspondence with the latitude-longitude projection system.

2.2. Weather radar data and basic settings. The radar data used in the paper are generated by a dual polarization Meteorological Doppler Weather Radar (MDWR) located at the Singapore Changi Airport - the eastern tip of the main island. A modern MDWR system generates hundreds of products related to meteorological conditions. In this paper, we use the standard Constant Altitude Plan Position Indicator (CAPPI) reflectivity data at $1 \mathrm{~km}$ above the mean sea level. The method can be applied to other CAPPI datasets at other user-definable heights. CAPPI reflectivity images contain information about the reflectivity (in units of $\mathrm{dBZ}$ ) at given grid points and times. These images are taken at 5-min intervals and 

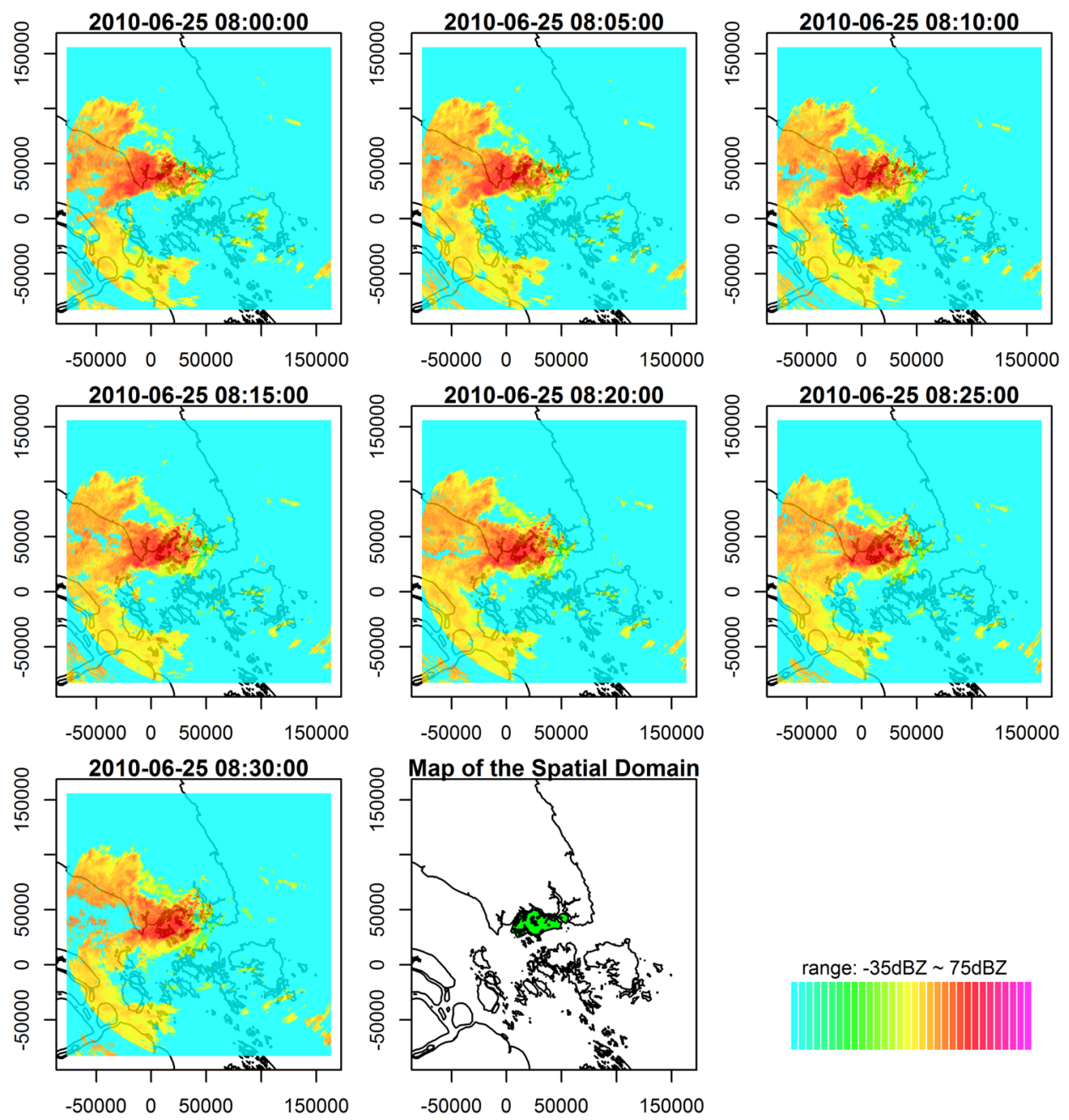

FIG. 1. Examples of radar scans of a tropical storm on the morning of 25 June 2010. The location of Singapore is indicated by the shaded area at the center of the last image, and the $x$-and $y$-coordinates are based on the SVY21 projection system, which has a one-to-one correspondence with the latitude-longitude projection system.

each image is arranged on a Cartesian 2D grid of $480 \times 480$ pixels, with the topleft and lower-right corners given by $\left(102.892^{\circ} \mathrm{E}, 2.42799^{\circ} \mathrm{N}\right)$ and $\left(105.052^{\circ} \mathrm{E}\right.$, $0.269748^{\circ} \mathrm{N}$ ), respectively. The resolution of each grid square is approximately $0.5 \times 0.5$ kilometers.

The $480 \times 480$ pixels of a radar image are further divided into $93 \times 93$ pixel arrays (i.e., boxes or tiles). Each pixel array has a fixed size of $19 \times 19$ pixels and covers an area of $90.25 \mathrm{~km}^{2}$. The centers of any two neighboring pixel arrays are spaced 5 pixels apart, which is approximately $2.5 \mathrm{~km}$. Figure 2(a) plots the 
(a)

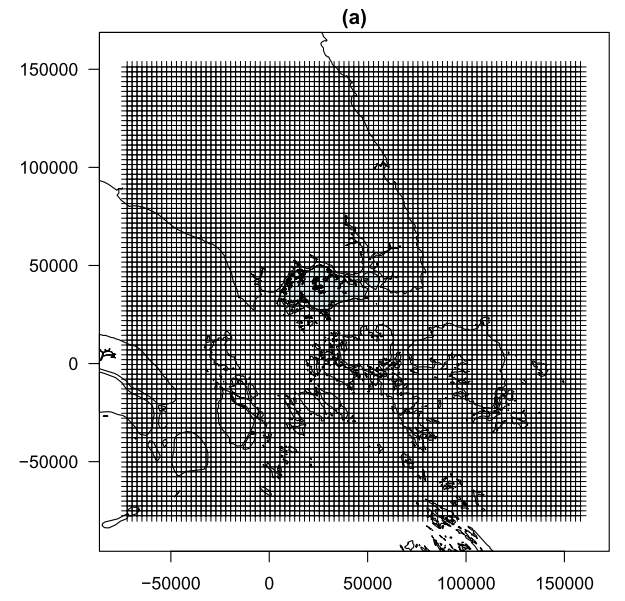

(b)

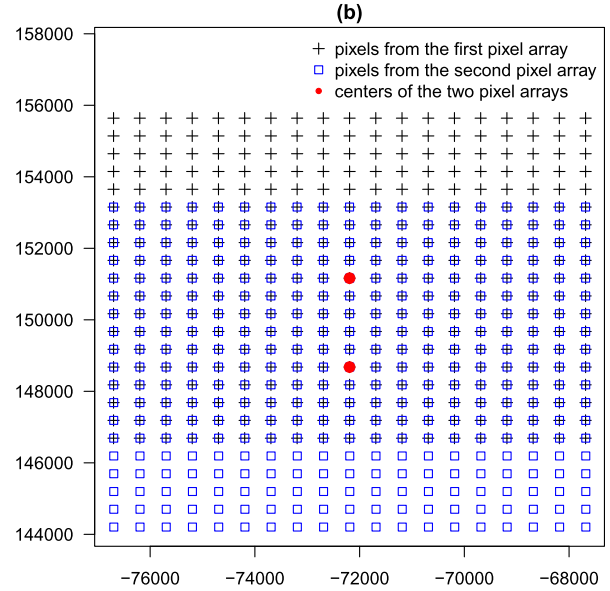

FIG. 2. (a) Centers of the 8649 pixel arrays occupying the spatial domain of a CAPPI image; (b) Illustration of two neighboring pixel arrays: each pixel array has a fixed size of $19 \times 19$ pixels and the centers of any two neighboring pixel arrays are spaced 5 pixels apart.

centers of the $93 \times 93$ pixel arrays that occupy the entire spatial domain of a radar scan. Note that, neighboring pixel arrays overlap with each other, as shown in Figure 2(b). This particular setting of pixel arrays is commonly adopted by the meteorological community [Li and Lai (2004)].

\section{The spatio-temporal modeling framework.}

3.1. A Lagrangian-type of advection scheme. Consider a Lagrangian-type of advection scheme within which an observer watches the world evolves around her as if she traveled with the pixel arrays. Let $\mathbb{S} \subseteq \mathbb{R}^{2}$ and $\mathbb{T} \subseteq \mathbb{N}^{+}$be the spatial and temporal domains that we denote by $x_{i, t}$ the location of pixel array $i$ at time $t, i=$ $1,2, \ldots, n$. Note that, pixel arrays are advected by a velocity field as the weather system moves, and we also denote by $s_{i}, i=1,2, \ldots, n$, the initial location of pixel array $i$ at some reference time $t=1$, that is, $x_{i, t}=s_{i}$ for all $i$ if $t=1$.

The velocity field characterizes the motion of pixel arrays. Since a twodimension problem is considered in this paper, the velocity field is the projection of the actual wind field on the surface $1 \mathrm{~km}$ above the sea level. Let $\mathbf{x}_{t}=\left(x_{1, t}, x_{2, t}, \ldots, x_{n, t}\right)^{T}$ be a collection of locations of all pixel arrays at time $t$. We define the forward translation (i.e., shift) operator $\Gamma$ as

$$
\Gamma \mathbf{x}_{t}=\left(\Gamma x_{1, t}, \Gamma x_{2, t}, \ldots, \Gamma x_{n, t}\right)^{T}=\mathbf{x}_{t+1}
$$

The translation operator $\Gamma$ can be determined given the velocity field estimated from a series of consecutive radar scans, and we leave the details to Appendix A. 
Based on equation (3.1), the $m$-step forward translation operator and inverse translation operator are also defined:

$$
\begin{aligned}
& \underbrace{\Gamma \Gamma \ldots \Gamma}_{m}=\Gamma^{(m)} x_{t}=\mathbf{x}_{t+m}, \quad m \in \mathbb{N}^{+}, \\
& \Gamma^{(-m)} \mathbf{x}_{t}=\mathbf{x}_{t-m}, \quad m \in \mathbb{N}^{+} .
\end{aligned}
$$

Similarly, let $\mathbf{Z}_{t}=\mathbf{Z}\left(\mathbf{x}_{t}\right)=\left(Z\left(x_{1, t}\right), Z\left(x_{2, t}\right), \ldots, Z\left(x_{n, t}\right)\right)^{T}$ denote the radar reflectivity on all pixel arrays at time $t$ with $Z\left(x_{i, t}\right)$ being the reflectivity on pixel array $i(i=1,2, \ldots, n)$. Then the classic forced-advection model is defined as [Staniforth and Cote (1991)]

$$
\frac{d \mathbf{Z}_{t}}{d t}=\frac{\partial \mathbf{Z}_{t}}{\partial t}+\mathbf{v}_{t} \cdot \nabla \mathbf{Z}_{t}=\mathbf{G}_{t}
$$

Here, $\mathbf{v}_{t}$ is the velocity field at time $t$ over the space. $\mathbf{G}_{t}=\mathbf{G}\left(\mathbf{x}_{t}\right)=\left(G\left(x_{1, t}\right)\right.$, $\left.G\left(x_{2, t}\right), \ldots, G\left(x_{n, t}\right)\right)^{T}$ is the forcing term that represents the growth (or decay) of radar reflectivity on pixel arrays at time $t$ with $G\left(x_{i, t}\right)$ being the reflectivity growth on pixel array $i$.

It is well known that there exists a discrete approximation to the forcedadvection problem (3.4) as follows:

$$
\begin{aligned}
\mathbf{Z}_{t+1} & =\mathbf{Z}\left(\mathbf{x}_{t+1}\right) \\
& =\mathbf{Z}\left(\Gamma^{(-2)} \mathbf{x}_{t+1}\right)+2 \mathbf{G}\left(\Gamma^{(-1)} \mathbf{x}_{t+1}\right)+O\left(\Delta^{2}\right) \\
& \approx \mathbf{Z}_{t-1}+2 \mathbf{G}_{t},
\end{aligned}
$$

where $\Delta$ is the length of time intervals.

The advection scheme (3.5) serves as the basis of our model, which suggests that the process $\left\{\mathbf{Z}_{t}\right\}$ is driven by two hidden sub-processes. The first sub-process is the dynamic velocity field which determines translation operator $\Gamma$, while the second is the reflectivity growth process $\left\{\mathbf{G}_{t}\right\}$. In other words, given the trajectory of any pixel array $i$, the reflectivity on that pixel array at time $t+1$ is the sum of the reflectivity on the pixel array at time $t-1$ and two times the reflectivity growth on the same pixel array at time $t$. Neither the velocity field (i.e., the trajectories of pixel arrays) nor the reflectivity growth are directly observed.

The velocity vector field of a weather system can be obtained by analyzing two consecutive radar scans. Once the velocity field has been established, the translation operators are fully defined and the locations of pixel arrays at different times can be tracked. Hence, the construction of the velocity field serves as a preliminary step for the entire modeling approach. As discussed in Section 1, we adopt a popular pattern-based method known by the meteorological community as the COTREC method (Tracking Radar Echoes by Correlation). This method has been well explained by Rinehart and Garvey (1978), Li, Schmid and Joss (1995) and Li and Lai (2004), and we provide a brief review of the principle of the method 
in Appendix A. In this paper, we treat the translation operators as known from the constructed velocity vector field. The error associated with the estimated velocity field is absorbed by the statistical model of $\left\{\mathbf{G}_{t}\right\}$ to be described.

Given the velocity field, it is possible to track the locations of pixel arrays at different times, $\left(\mathbf{x}_{1}, \mathbf{x}_{2}, \ldots, \mathbf{x}_{t}\right)$, and obtain the observed reflectivity $\left(\mathbf{Z}_{1}, \mathbf{Z}_{2}, \ldots, \mathbf{Z}_{t}\right)$ associated with the tracked pixel arrays from radar images. Then the reflectivity growth process $\left\{\mathbf{G}_{t}\right\}$ is obtained from the Lagrangian-type of advection scheme (3.5):

$$
\mathbf{G}_{t}=\mathbf{G}\left(\Gamma^{(-1)} \mathbf{x}_{t+1}\right)=\frac{\mathbf{Z}\left(\mathbf{x}_{t+1}\right)-\mathbf{Z}\left(\Gamma^{(-2)} \mathbf{x}_{t+1}\right)}{2}
$$

In other words, the reflectivity growth is exactly the mismatch between two radar scans under the velocity vector field [Radhakrishna, Zawadzki and Fabry (2012), Germann and Zawadzki (2002)]. In a special case in which pixel arrays do not move, the reflectivity growth is immediately obtained from the difference of reflectivity from two consecutive radar scans. Of course, this is never the case in reality when modeling radar reflectivity data. In the next subsection, we describe how the reflectivity growth process $\left\{\mathbf{G}_{t}\right\}$ is modeled.

3.2. The modeling. Extrapolating radar reflectivity is the mainstay of nowcasting. In Singapore, for example, the official lead time for the warnings of heavy storms is usually between 15 and 45 minutes. Since only radar reflectivity at a fixed height is used in this paper, it is a natural choice to consider the (possibly transformed) reflectivity growth process $\left\{\mathbf{G}_{t}\right\}$ as an Autoregressive (AR) process. In particular, we assume that the process $\left\{\mathbf{G}_{t}\right\}$ has the following general form:

$$
\mathbf{B}_{t} \mathbf{G}_{t}=r_{1} \mathbf{B}_{t-1} \mathbf{G}_{t-1}+r_{2} \mathbf{B}_{t-2} \mathbf{G}_{t-2}+\cdots+r_{q} \mathbf{B}_{t-q} \mathbf{G}_{t-q}+\varepsilon_{t},
$$

where $\left(r_{1}, r_{2}, \ldots, r_{q}\right)$ are the autoregression coefficients, $\varepsilon_{t}$ is some spatiotemporal error process, $q$ is the order of the AR process and the matrix $\mathbf{B}$ is to be determined for each time point.

At any time $t$, we model $\mathbf{G}_{t}$ as a spatio-temporal process and assume that $\mathbf{G}_{t}$ can be expressed by

$$
\mathbf{G}_{t}=\mu_{t}+\mathbf{Y}_{t}
$$

where $\mu_{t}=\left(\mu_{1, t}, \mu_{2, t}, \ldots, \mu_{n, t}\right)^{T}$ captures the deterministic large-scale spatial trend of the reflectivity growth at time $t$ with its element $\mu_{i, t}$ representing the mean reflectivity growth of pixel array $i$, and $\mathbf{Y}_{t}$ describes the small-scale random variation of the reflectivity growth with zero-mean and spatial covarance $\boldsymbol{\Sigma}_{t}$.

In particular, the mean function $\mu_{t}$ needs to be flexible enough to handle the complex reflectivity growth over the spatial domain. Hence, we consider a locally weighted mixture of linear regression model as shown below [Stroud, Müller and Sansó (2001)]:

$$
\mu_{i, t}=\sum_{j=1}^{J} \pi_{j}\left(x_{i, t}\right) \mathbf{f}_{j}^{T}\left(x_{i, t}\right) \gamma_{j, t}
$$


where $\mathbf{f}_{j}\left(x_{i, t}\right)$ is a column vector of known basis functions, $\gamma_{j, t}$ are column vectors of unknown parameters at time $t$, and $\pi_{j}$ is a nonnegative kernel centered at chosen locations. Let $\mathbf{F}_{j, t}=\left(\mathbf{f}_{j}\left(x_{1, t}\right), \mathbf{f}_{j}\left(x_{2, t}\right), \ldots, \mathbf{f}_{j}\left(x_{n, t}\right)\right)$ and $\pi_{j}=$ $\left(\pi_{j}\left(x_{1, t}\right), \pi_{j}\left(x_{2, t}\right), \ldots, \pi_{j}\left(x_{n, t}\right)\right)$, we have

$$
\mu_{t}=\mathbf{F}_{t} \gamma_{t}
$$

where $\mathbf{F}_{t}=\left(\operatorname{diag}\left(\pi_{1}\right) \mathbf{F}_{1, t}, \operatorname{diag}\left(\pi_{2}\right) \mathbf{F}_{2, t}, \ldots, \operatorname{diag}\left(\pi_{J}\right) \mathbf{F}_{J, t}\right)$ and $\gamma_{t}=\left(\gamma_{1, t}^{T}, \gamma_{2, t}^{T}\right.$, $\left.\ldots, \gamma_{J, t}^{T}\right)^{T}$.

The second term of (3.8), $\mathbf{Y}_{t}$, is modeled by an STCAR model to handle both the spatial and temporal association among pixel arrays. For any pixel array $i$ and given $\left\{y\left(x_{j, t}\right) ; j \neq i\right\}$ on all pixel arrays $j$ which belongs to a pre-defined neighborhood $\Omega_{i}$ of $i$ (i.e., $j \in \Omega_{i}$ and $j \neq i$ ), the conditional distribution of $Y\left(x_{i, t}\right)$ is a Gaussian given as follows:

$$
Y\left(x_{i, t}\right) \mid\left\{Y\left(x_{j, t}\right) ; j \neq i, j \in \Omega_{i}\right\} \sim N\left(\rho_{t} \sum_{j \neq i} \frac{w_{i, j}(t)}{w_{i+}(t)} y\left(x_{j, t}\right), \sigma_{i, t}^{2}\right),
$$

where

$$
w_{i, j}(t)= \begin{cases}\phi\left(x_{i, t}, x_{j, t}\right) & \text { if } j \in \Omega_{i}, i \neq j, \\ 0 & \text { otherwise, }\end{cases}
$$

and $w_{i+}(t)=\sum_{j}^{n} w_{i, j}(t)$, and $\phi$ is a nonincreasing function of the distance between $x_{i, t}$ and $x_{j, t}$. In (3.11), the parameter $\sigma_{i, t}$ describes the variability of the data at location $x_{i, t}$, and the parameter $\rho_{t}$ represents the strength of spatial association at time $t$.

Note that the distance between two pixel arrays changes over time. If the neighborhood of a pixel array $i$ is defined in a conventional way as a set of pixel arrays within a fixed distance of array $i$, then the number of pixel arrays in a neighborhood varies over time as the pixel arrays travel in space. This is mathematically inconvenient for an autoregressive model like (3.11). Fortunately, within a short period of time (say, 10 to 15 minutes), it is reasonable to assume that neighboring pixels tend to move in a similar direction with a similar speed, provided that the wind field is smooth and slowly varying in space. As shown in the numerical example presented in Section 4, the order of $q$ of the AR process (3.7) is typically 2 to 3 , which corresponds to 10 to 15 minutes. Based on the above considerations, we define the neighborhood of a pixel array $i$ based on the initial locations of pixel arrays at time $t=1$ as follows:

$$
\Omega_{i}=\left\{j \in \mathbb{S}:\left\|x_{i, 1}-x_{j, 1}\right\|<d\right\}
$$

for a constant distance $d>0$.

Let $\mathbf{W}_{t}=\left\{w_{i, j}(t)\right\}_{i, j=1}^{n}, \mathbf{W}_{D, t}=\operatorname{diag}\left(w_{1+}(t), w_{2+}(t), \ldots, w_{n+}(t)\right)$ and $\sigma_{i, t}=$ $\sigma_{t} \cdot w_{i+}^{-1}(t), \mathbf{Y}_{t}$ is modeled as a temporal sequence of Conditional Autoregressive (CAR) models with expected value zero, that is,

$$
\mathbf{Y}_{t} \sim N\left(\mathbf{0}, \sigma_{t}^{2}\left(\mathbf{W}_{D, t}-\rho_{t} \mathbf{W}_{t}\right)^{-1}\right) .
$$


Then the vector of random fields, $\mathbf{Y}_{t}$, is an STCAR model of order $q$ if for every $t$,

$$
\mathbf{B}_{t} \mathbf{Y}_{t}=\sum_{j}^{q} r_{j} \mathbf{B}_{t-j} \mathbf{Y}_{t-j}+\varepsilon_{t}
$$

where

$$
\begin{aligned}
\varepsilon_{t} & \sim N\left(\mathbf{0}, \sigma_{t}^{2} \mathbf{W}_{D, t}^{-1}\left(\mathbf{I}-\rho_{t} \mathbf{W}_{D, t}^{-1} \mathbf{W}_{t}\right)^{T}\right), \\
\mathbf{B}_{t} & =\mathbf{I}-\rho_{t} \mathbf{W}_{D, t}^{-1} \mathbf{W}_{t} .
\end{aligned}
$$

To ensure that the covariance matrix $\boldsymbol{\Sigma}_{t}$ is positive definite, $\rho_{t}$ needs to be between the interval $\left(1 / \lambda^{\max }, 1 / \lambda^{\min }\right)$ with $\lambda^{\min }$ and $\lambda^{\max }$, respectively, the maximum and minimum eigenvalues of the matrix $\mathbf{W}_{D, t}^{-1} \mathbf{W}_{t}$ [Haining (1990)].

Substituting (3.8) and (3.10) into (3.7), we have

$$
\mathbf{B}_{t} \mathbf{G}_{t}=\sum_{j=1}^{j=q} r_{j} \mathbf{B}_{t-1} \mathbf{F}_{t-j} \gamma_{t-j}+\sum_{j=1}^{j=q} r_{j} \mathbf{B}_{t-j} \mathbf{Y}_{t-j}+\varepsilon_{t} .
$$

Hence, (3.18) suggests that the reflectivity growth process $\left\{\mathbf{G}_{t}\right\}$ is modeled by an STCAR with order $q$. The first term on the right-hand side of (3.18) describes the mean process, while the second term on the right of (3.18) is an STCAR model defined by (3.15).

The autoregressive nature of the model (3.18) allows us to extrapolate the radar images for nowcasting purposes. At any time $t^{\prime}$, the one-step-ahead reflectivity $Z_{t^{\prime}+1}$ can be predicted by (3.5), that is, $\mathbf{Z}_{t^{\prime}+1}=\mathbf{Z}_{t^{\prime}-1}+2 \mathbf{G}_{t^{\prime}}$. Here, the reflectivity $\mathbf{Z}_{t^{\prime}-1}$ at $t^{\prime}-1$ is observed, and $\mathbf{G}_{t^{\prime}}$ is estimated from (3.18). In particular,

$$
\mathbf{G}_{t^{\prime}} \sim N\left(\sum_{j=1}^{q} r_{j} \mathbf{B}_{t^{\prime}}^{-1} \mathbf{B}_{t^{\prime}-j} \mathbf{G}_{t^{\prime}-j}, \sigma_{t^{\prime}}^{2}\left(\mathbf{W}_{D, t^{\prime}}-\rho_{t^{\prime}} \mathbf{W}_{t^{\prime}}\right)^{-1}\right) .
$$

Similarly, the reflectivity fields at times $t^{\prime}+2, t^{\prime}+3, \ldots, t^{\prime}+p$ are obtained iteratively.

3.3. Parameter estimation. The proposed STCAR model contains a set of unknown parameters, including a $3 J \times 1$ column vector, $\gamma_{t}=\left(\gamma_{1, t}^{T}, \gamma_{2, t}^{T}, \ldots, \gamma_{J, t}^{T}\right)^{T}$, that determines the overall spatial trend of the reflectivity growth at time $t$, the spatial association $\rho_{t}$ at time $t$, the variability $\sigma_{t}$ at time $t$, and the parameters of the temporal association, $\mathbf{r}=\left(r_{1}, r_{2}, \ldots, r_{q}\right)$.

Hence, a large number of unknown parameters is to be estimated for any time $t$. In the application example presented in the next section, for example, the number of kernels $J$ is chosen to be 30 , which makes $\gamma_{t}$ a $90 \times 1$ column vector. In practice, a new radar image becomes available every 5 minutes. Considering the time consumed by the preliminary radar data processing and the construction of 
velocity field, it is of vital importance for the parameter estimation procedure to be numerically stable and efficient enough to incorporate the latest information into the analysis. This motivates us to adopt a two-step estimation approach based on the idea described in Mariella and Tarantino (2010). In the first step, we ignore the temporal association between radar images, and estimate the parameters $\gamma_{t}, \rho_{t}$, and $\sigma_{t}$ from individual radar images. In the second step, the temporal association $\mathbf{r}=\left(r_{1}, r_{2}, \ldots, r_{q}\right)$ is estimated based on the results from the first step.

In particular, the parameters $\gamma_{t}, \rho_{t}$, and $\sigma_{t}$ are estimated using the Iteratively Re-Weighted Generalized Least Squares (IRWGLS). Note that, when the temporal association of radar images is ignored, (3.18) degenerates to a conventional CAR model without temporal correlation between a sequence of radar scans:

$$
\mathbf{B}_{t} \mathbf{G}_{t}=\mathbf{F}_{t} \gamma_{t}+\varepsilon_{t}, \quad \varepsilon_{t} \sim N\left(\mathbf{0}, \sigma_{t}^{2} \mathbf{W}_{D, t}^{-1}\left(\mathbf{I}-\rho_{t} \mathbf{W}_{D, t}^{-1} \mathbf{W}_{t}\right)^{T}\right)
$$

The IRWGLS consists of the following steps:

Step 0: Set the initial $\hat{\boldsymbol{\Sigma}}_{t}$ to an identify matrix.

Step 1: Estimate $\gamma_{t}$ using the Feasible General Least Squares (FGLS):

$$
\hat{\gamma}_{t}=\left(\mathbf{F}_{t}^{\top} \hat{\mathbf{\Sigma}}_{t}^{-1} \mathbf{F}_{t}\right)^{-1} \mathbf{F}_{t}^{\top} \hat{\mathbf{\Sigma}}_{t} \mathbf{G}_{t}
$$

Step 2: Based on the residuals $\mathbf{u}=\mathbf{G}_{t}-\mathbf{F}_{t} \hat{\gamma}_{t}$, estimate the spatial association $\rho_{t}$ and variability $\sigma_{t}$ using the Maximum Likelihood Estimation (MLE) described below, and obtain the estimate of the covariance matrix, $\hat{\mathbf{\Sigma}}_{t}$, from equation (3.14).

Step 3: Iterate Steps 1 and 2 until the relative changes of $\hat{\gamma}_{t}, \hat{\rho}_{t}$, and $\hat{\sigma}_{t}$ are small. In the first iteration, since $\hat{\boldsymbol{\Sigma}}_{t}$ is an identify matrix, $\hat{\gamma}_{t}$ in Step 1 is the Ordinary Least Squares (OLS) estimator and is unbiased. In subsequent iterations, the finite-sample properties of the FGLS estimator, $\hat{\gamma}_{t}$, are usually unknown. Asymptotically, the FGLS estimator possesses the asymptotic properties of the Maximum Likelihood estimator, and is equivalent to the Generalized Least Squares (GLS) estimator under regularity conditions. In fact, it is possible to obtain estimate $\gamma_{t}, \rho_{t}$, and $\sigma_{t}$ all at once using MLE. However, this leads to a high-dimensional optimization problem which could be numerically inefficient in practice.

In step 2 above, given the observed reflectivity $\mathbf{z}_{t-1}$ and $\mathbf{z}_{t+1}$, the log-likelihood function is given as follows:

$$
l\left(\mathbf{y}_{t} ; \gamma_{t}, \sigma_{t}, \rho_{t}\right)=-\frac{n}{2} \log \left(2 \pi \sigma_{t}^{2}\right)+\frac{1}{2} \log \left|\mathbf{W}_{D, t} \mathbf{B}_{t}\right|-\frac{\mathbf{y}_{t}^{T} \mathbf{W}_{D, t} \mathbf{B}_{t} \mathbf{y}_{t}}{2 \sigma_{t}^{2}}
$$

where

$$
\mathbf{y}_{t}=\mathbf{g}_{t}-\mathbf{F} \gamma_{t},
$$

and $\mathbf{g}_{t}=\left(\mathbf{z}\left(\mathbf{x}_{t+1}\right)-\mathbf{z}\left(\Gamma^{(-2)} \mathbf{x}_{t+1}\right)\right) / 2$. 
Note that, the MLE of $\sigma_{t}^{2}$ is given by

$$
\hat{\sigma}_{t}^{2}=\frac{\mathbf{y}_{t}^{T} \mathbf{W}_{D, t} \mathbf{B}_{t} \mathbf{y}_{t}}{n} .
$$

Substituting (3.23) into the log-likelihood function (3.21), the MLE of $\gamma_{t}$ and $\rho_{t}$ can be numerically found by minimizing

$$
\frac{n}{2} \log \left(\frac{\mathbf{y}_{t}^{T} \mathbf{W}_{D, t} \mathbf{B}_{t} \mathbf{y}_{t}}{n}\right)-\frac{1}{2} \log \left|\mathbf{W}_{D, t} \mathbf{B}_{t}\right| .
$$

Multiple initial values can be used to avoid the convergence to local minima, given the high dimension of the optimization problem.

After the parameters $\gamma_{t}, \rho_{t}$, and $\sigma_{t}$ have been estimated, it is possible to estimate the temporal association parameter $\mathbf{r}=\left(r_{1}, r_{2}, \ldots, r_{q}\right)$. Note that, (3.18) degenerates to a conventional linear regression model (3.25) without spatial correlation between the reflectivity on pixel arrays if $\rho_{t}=0$ for all $t$. Hence, $\mathbf{r}$ can be estimated using the weighted least squares after substituting $\left(\hat{\gamma}_{t}, \hat{\sigma}_{t}, \hat{\mathbf{W}}_{D, t}, \mathbf{y}_{t}\right)$ into (3.25):

$$
\mathbf{G}_{t}=\sum_{j=1}^{j=q} r_{j} \mathbf{F}_{t-j} \hat{\gamma}_{t-j}+\sum_{j=1}^{j=q} r_{j} \mathbf{Y}_{t-j}+\varepsilon_{t},
$$

where $\varepsilon_{t} \sim N\left(\mathbf{0}, \hat{\sigma}_{t}^{2} \hat{\mathbf{W}}_{D, t}^{-1}\right)$. Note that, $\mathbf{B}_{t}$ becomes an identity matrix if $\rho_{t}=0$.

4. Application example. We revisit the motivating example presented in Section 2, and apply the proposed modeling approach to the radar images, shown in Figure 1, on the early morning of 25 June 2010.

4.1. Obtain the velocity field. The first step is to construct the velocity field, making it possible to track pixel arrays as well as the reflectivity change on those arrays. The basic settings of the overlapped pixel arrays are described in Section 2 and visualized in Figure 2. For illustrative purposes, Figure 3 shows the constructed velocity fields at 8:00 AM, which is the starting time of the sequence of radar images shown in Figure 1. The velocity fields are obtained using two consecutive radar scans. In order to visualize the velocity vectors clearly, we only show the constructed velocity fields over Singapore, that is, the central part of the spatial domain. The moving directions of pixel arrays are indicated by arrows with their length proportional to the moving speed. Note that, since the velocity vectors are obtained based on the Pearson's correlation coefficient of the reflectivity values on pixel arrays from two consecutive scans, it is only possible to obtain the velocity vectors for areas where the weather system is located.

To further illustrate the pixel tracking method, the left panel of Figure 4 shows the initial location of a pixel array at 8:00 AM. The right panel of Figure 4 shows the tracked locations of this pixel array at different times. This pixel array heads 
2010-06-25 08:00:00 (zoomed-in)
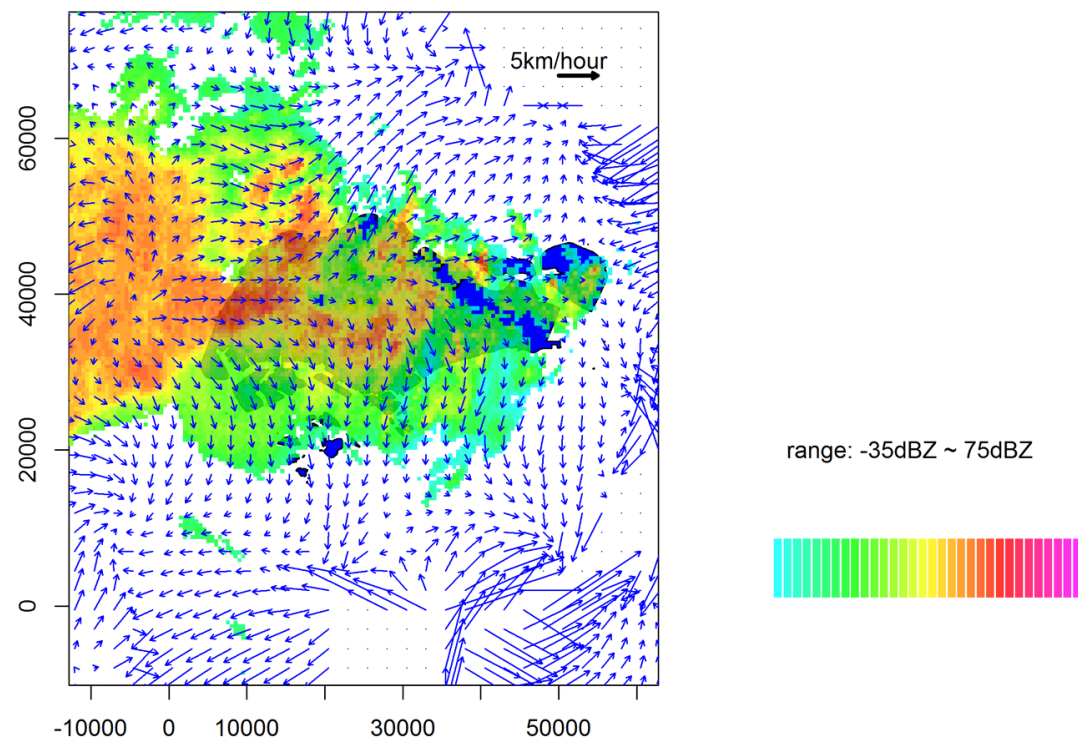

FIG. 3. The constructed velocity field at 8:00 AM over Singapore (the shaded area in the center). The arrows indicate the wind direction and the length of the arrow indicate exactly the wind speed. Only positive reflectivity ( $>0 \mathrm{dBZ}$ ) is plotted to clearly show the wind vectors, although the wind field is obtained using the complete reflectivity data.

to the east at the beginning, and then turns to the north direction at 8:30 AM. Figure 5 also shows the observed reflectivity on this tracked pixel array (with 361 pixels) at different times. Recall that the pixel arrays are tracked by maximizing
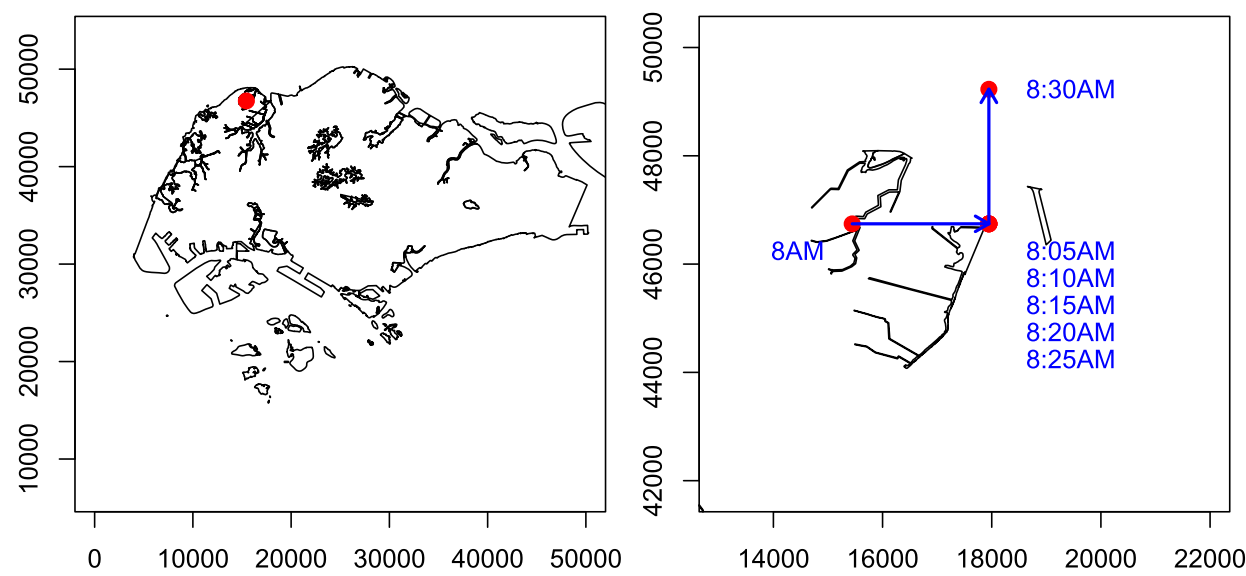

FIG. 4. Illustration of the tracking of pixel arrays. The left panel shows the initial location of a pixel array, and the right panel shows the tracked locations of this pixel array at different time. 
08:00:00

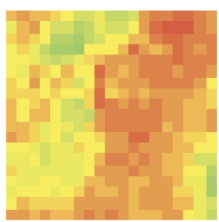

08:20:00

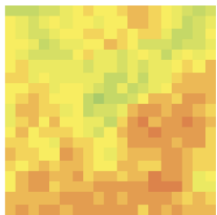

08:05:00

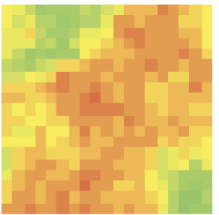

08:25:00

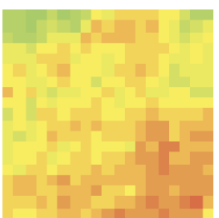

08:10:00

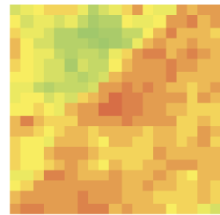

08:30:00

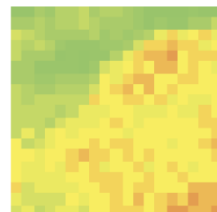

08:15:00
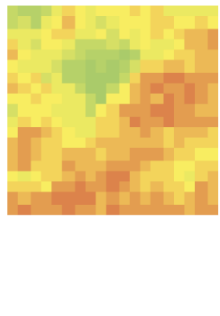

FIG. 5. Reflectivity image on the tracked pixel array at different times.

the cross-correlation between areas from two consecutive radar scans, and it is possible to see the similarity of the seven images. Although the 6th image (taken at 8:25 AM) and the 7th image (taken at 8:30 AM) appear less similar, the correlation between the 6th image at 8:25 AM and any other pixel arrays at time 8:30 AM is even weaker. In general, it is difficult to construct the velocity vectors when the underlying weather system is rapidly changing over time. Fortunately, to enhance the robustness of the tracking algorithm, the smoothness/consistency constraint is incorporated in the constrained minimization problem (A.4) in Appendix A. Such a constraint ensures that neighboring pixel arrays move in similarly directions, which effectively prevents a single velocity vector from being estimated incorrectly.

4.2. Model the reflectivity field. Once the velocity field has been established, the reflectivity growth, $\mathbf{G}_{t}$, is obtained using (3.6) and shown in Figure 6. As one might expect, high reflectivity growth is found at the central area of the storm where the weather system is highly dynamic, while the reflectivity approximately remains unchanged over the 5-min sampling interval for most pixel arrays.

The model parameters $\rho$ (the overall spatial association), $\sigma$ (the overall spatial variability) and $r$ (the temporal association) are estimated using the method described in Section 3.3. In particular, we place a number of $J=30$ Gaussian kernels over the reflectivity field with the centers chosen by the method of K-means clustering [Hartigan and Wong (1979)]. For each Gaussian kernel, the covariance matrix is chosen as a diagonal matrix with standard deviation $10 \mathrm{~km}$. Table 1 shows the estimated $\rho$ and $\sigma$ from 8:05 AM to 8:30 AM. 

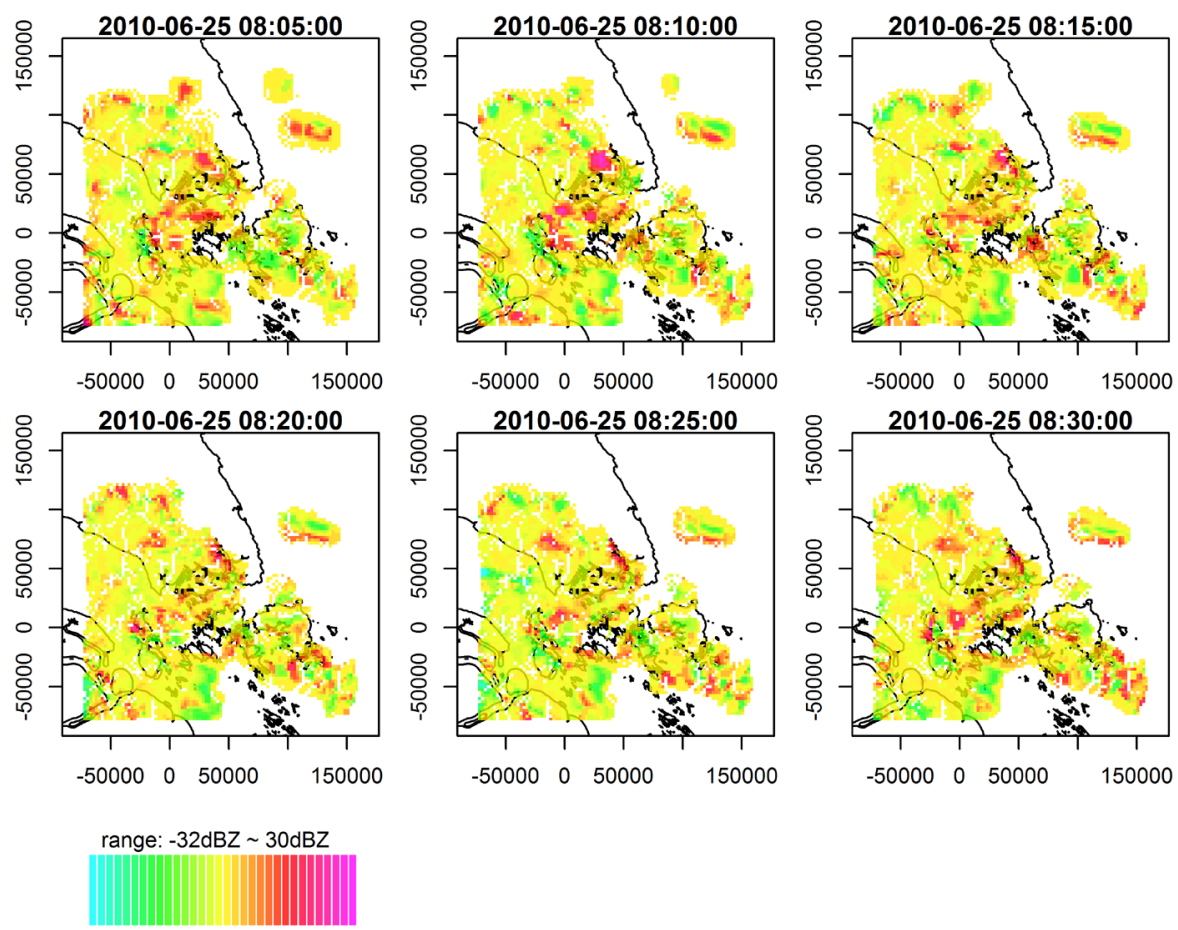

FIG. 6. Reflectivity growth over the spatial domain from 8:05 AM and 8:30 AM.

As seen from Table 1 , the values of $\sigma$, which characterizes the overall variability, does not change dramatically over the 25-min interval from 8:05 AM to 8:30 AM. The spatial association $\rho$, however, jumps from -11.63 at 8:05 AM to -19.22 at 8:10 AM and remains around -19 after that. Substituting the estimated values from Table 1 into (3.14), we obtain the estimated covariance matrix $\hat{\boldsymbol{\Sigma}}_{t}$, which allows us to further investigate the correlation structure of the reflectivity growth among neighboring pixel arrays. Figure 7 plots the computed correlation between all pairs of pixel arrays against their distances in kilometer. In particular, the left panel shows the correlation at 8:05 AM where $\rho$ is close to -11 , while the right panel corresponds to 8:30 AM where $\rho$ is close to -19 . Interestingly, the two plots suggest very different correlation structures. At 8:05 AM, the neighbor-

TABLE 1

Estimated values of $\rho$ and $\sigma$ from 8:05 AM to 8:30 AM

\begin{tabular}{rrrrrrr}
\hline & 8:05 AM & 8:10 AM & 8:15 AM & 8:20 AM & 8:25 AM & 8:30 AM \\
\hline$\rho$ & -11.63 & -19.22 & -18.96 & -19.12 & -19.19 & -19.32 \\
$\sigma$ & 18.67 & 16.20 & 16.34 & 15.84 & 17.44 & 17.08 \\
\hline
\end{tabular}



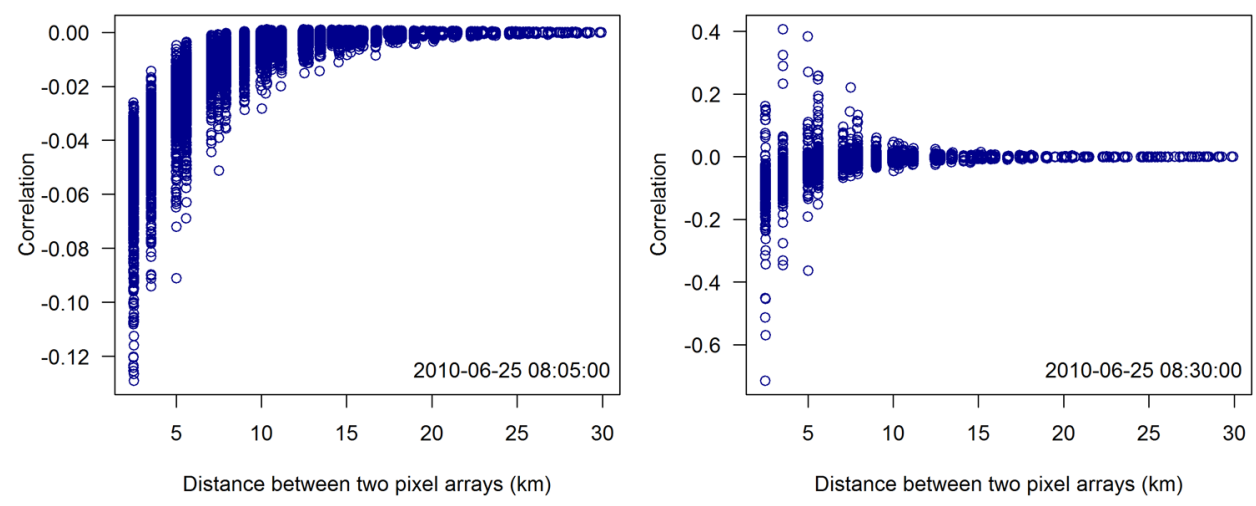

FIG. 7. Correlation between all pairs of pixel arrays.

ing pixel arrays are negatively correlated. For two pixel arrays which are close to each other, some pairs of pixel arrays exhibits stronger correlation (say, -0.12) than others (say, 0.02). In general, the correlation becomes weaker as the distance between two pixel arrays grows and can be ignored when two pixel arrays are $30 \mathrm{~km}$ apart. At 8:30 AM, the correlation structure among pixel arrays seems to be more complicated. Not only the strength of correlation becomes much stronger $(-0.6 \sim 0.4)$, but also the neighboring pixel arrays could either exhibits positive or negative correlation. Such a puzzling result concerning the correlations implied by the CAR model has been studied by Wall (2004) and Assunção and Krainski (2009). The authors found that, when $\rho$ increases from zero to the upper bound of its parameter space, the correlation is positive and monotone increasing as $\rho$. When $\rho$ decreases from zero, the correlation is negative at first and also decreases as $\rho$. But, when $\rho$ is further approaching to the lower bound of its parameter space, the correlation between some neighbors could either approaches -1 or starts growing to the positive side. In our context, the negative correlation among two neighboring pixel arrays might be explained by the mass conservation as the water contained in the cloud leaving one region and joining its neighboring regions. The positive correlation is often expected as the reflectivity in a particular region is growing or decaying at the same time.

Once $\hat{\gamma}_{t}, \hat{\sigma}_{t}$ and $\hat{\mathbf{W}}_{D, t}$ have been estimated, $\mathbf{r}$ is obtained from (3.25) using the weighted least squares. If the order of the STCAR model is chosen to be $q=$ $2,3,4$, we respectively obtain $\hat{\mathbf{r}}=(0.911,-0.317), \hat{\mathbf{r}}=(0.996,-0.495,0.21)$, and $\hat{\mathbf{r}}=(1.082,-0.513,0.347,-0.206)$. The autoregressive nature of (3.18) allows us to extrapolate the radar images for nowcasting purposes. The reflectivity field at 8:35 AM (i.e., 5-minutes-ahead nowcasting) is predicted using (3.19) and is shown in Figure 8. Similarly, the reflectivity fields at 8:45 AM (i.e., 15minutes-ahead) and 9:00 AM (i.e., 30-minutes-ahead) are also obtained iteratively and shown in Figure 8. In this figure, the first row shows the actually observed reflectivity at 8:35 AM, 8:45 AM and 9:00 AM, the second rows shows the predicted 

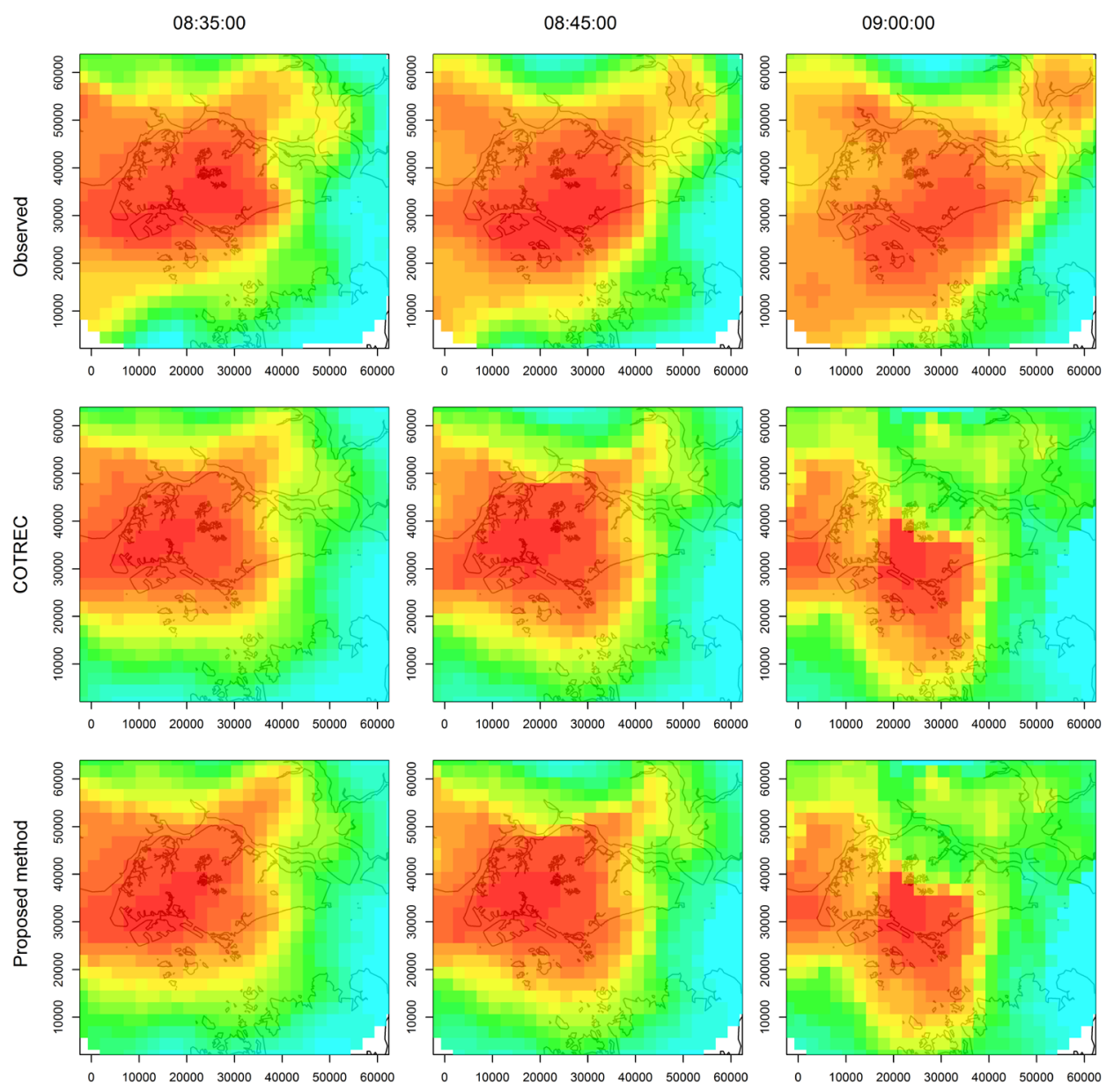

FIG. 8. Observed and predicted reflectivity fields at 8:35 AM, 8:45 AM, and 9:00 AM. The first row shows the actually observed reflectivity, the second rows shows the predicted reflectivity using the existing COTREC method, and the third row shows the predicted reflectivity using the proposed approach.

reflectivity using the existing COTREC method without considering the grow and decay of reflectivity [Li and Lai (2004)], and the third row shows the predicted reflectivity using the proposed approach.

The COTREC method is a well-known approach which has been widely implemented in the meteorological community [Li and Lai (2004), RMI (2008)]. While both the COTREC and the proposed approaches rely on the same patternbased method to construct the wind field, the former (which is not a statistical model) does not model the growth and decay of the reflectivity as the proposed method does. Hence, the COTREC method serves as an ideal candidate for comparison purposes. It is seen from Figure 8 that the reflectivity fields generated by 
both methods reasonably match with the actually observed reflectivity fields for 5-min- and 15-min-ahead nowcasting. However, the 30-min-ahead nowcasting at 9:00 AM becomes less accurate. At 9:00 AM, the actual reflectivity field heads to the east, while the predicted high reflectivity region moves to the south. Since the radar-based nowcasting method is based on the spatio-temporal extrapolation (autoregression in our case) of radar reflectivity, it is almost impossible to predict the change of the wind direction for highly dynamic tropical storms. Note that the constructed velocity vectors associated with the high reflectivity region at the center of the spatial domain (as shown by Figure 3) are pointing to the south at 8:30 AM. In general, the predicability of the same radar-based nowcasting method varies with geo-locations [Radhakrishna, Zawadzki and Fabry (2012)]. In tropical areas where storm systems are highly dynamic and embedded with numerous small-scale convective cells, which change rapidly within tens of minutes, the radar-based nowcasting methods usually provide reasonable quantitative prediction within a very short period of time. For longer period prediction, it is a common practice in the meteorological community to incorporate the downscaled output generated by computationally-intensive numerical weather prediction models, such as the Weather Research and Forecast (WRF) model, through data assimilation [Bowler, Pierce and Seed (2006), Seed, Pierce and Norman (2013)].

To further compare the performance between the COTREC and the proposed method, Figure 9 shows the accumulative mean-squared-error of the predicted reflectivity at pixel arrays. Note that we focus on the accumulative MSE rather than the MSE at each time point because precipitation nowcasting in practice is often concerned with the prediction of the accumulative amount up to a certain time point. The left panel shows the comparison for all pixel arrays. In addition, since
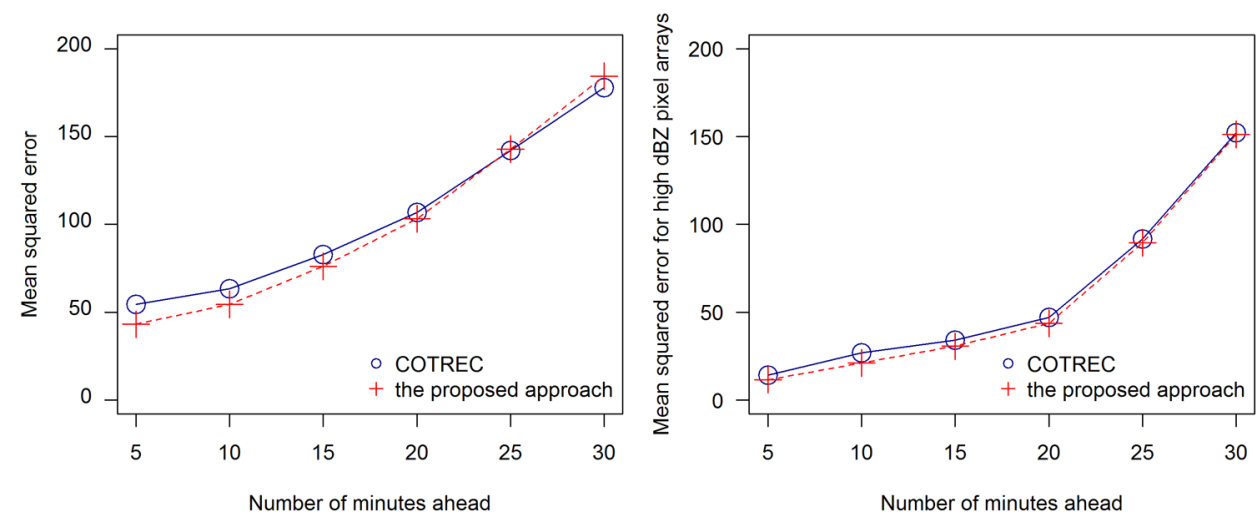

FIG. 9. Comparison of MSE between the existing COTREC method and the proposed method for the storm on 25 June 2010. The left panel shows the MSE computed from all pixel arrays, and the right panel shows the MSE computed only from those pixel arrays of interest with reflectivity greater than $35 \mathrm{dBZ}$. 
only the pixel arrays with high reflectivity are of interest, the right panel shows the comparison for pixel arrays with reflectivity greater than $35 \mathrm{dBZ}$. Empirically, $35 \mathrm{dBZ}$ corresponds to $5.6 \mathrm{~mm} / \mathrm{hr}$ or $0.22 \mathrm{in} / \mathrm{hr}$ moderate rains. We see that it is worth considering the growth and decay of reflectivity for short-term nowcasting. As seen from the left panel of Figure 9, the proposed method outperforms if the nowcasting horizon is not greater than 25 minutes. If we only focus on pixel arrays with reflectivity greater than $35 \mathrm{dBZ}$, the proposed method outperforms up to 30-min-ahead nowcasting. As we have already discussed, the reflectivity growth is extrapolated through a space-time autoregression model, the performance of such a model will deteriorate as the prediction horizon increases for extremely dynamic tropical storms. Hence, our findings suggest that the gain of incorporating the growth and delay into the model becomes zero or even negative when the prediction horizon is beyond what the model can offer. The cut-off threshold varies with geo-locations, seasons and types of precipitation, and can be found in practice through numerical experiments on historical data. When cumulative rainfall is of interest in practice, the predicted rainfall amount is sensitive even to a small improvement in reflectivity prediction, especially for heavy rainfall events. Based on the Marshall-Palmer relationship, for example, $45 \mathrm{dBZ}$ usually corresponds to $0.92 \mathrm{in} / \mathrm{h}$ moderate to heavy rain, while $50 \mathrm{dBz}$ corresponds to $1.9 \mathrm{in} / \mathrm{h}$ heavy rain.

5. Comparison and discussions. In this section, the proposed method is applied to a number of 276 tropical storms recorded in 2010 and 2011. Comparison studies are then performed between the proposed method and the COTREC method described in the previous section. Table 2 provides a summary of the storm events used in the comparison study. Among the 276 storm events, a number 77 events are observed from the Inter-Monsoon Season (April-May and OctoberNovember), while the remaining 199 storms are observed in the Southwest Monsoon Season (June-September). Tropical storms behave in fundamentally different ways in these two seasons, leading to different findings and important operational insights of the proposed method, as described below.

5.1. Inter-monsoon season events. We first present the comparison results based on the 77 storms from the Inter-Monsoon Season. The Inter-Monsoon Season (April-May and October-November) in Singapore experiences showers in the

TABLE 2

A Summary of the Tropical Storms in the Comparison Study

\begin{tabular}{lccc}
\hline Season & Months & Number of events & Type of storms \\
\hline Inter-Monsoon & Apr-May and Oct-Nov & 77 & Small-scale; localized convective \\
Southwest Monsoon & Jun-Sep & 199 & Large-scale; persistent \\
\hline
\end{tabular}


afternoons and early evenings. As discussed in Section 1.2, the most common type of thunderstorms in the Inter-Monsoon Season are known as the small-scale localized convective weather system. For such a weather system, heavy thunderstorms can develop, grow, and dissipate very suddenly at a relatively small scale in a random manner, and the exact physics behind the convective processes which lead to the formation of such thunderstorms is not yet fully understood. The method developed in this paper is particularly designed to capture the such small scale features by modeling the reflectivity growth and decay for each pixel array using spatio-temporal statistical methods.

Figure 10 shows the box plot of the Mean Squared Error (MSE) associated with the extrapolated radar reflectivity fields based on the 77 storm events observed from the Inter-Monsoon season. The horizontal axis shows the nowcasting horizon, which ranges from 5 minutes to 30 minutes. Similar to Figure 9, the left panel shows the MSE evaluated based on all pixel arrays in a radar image. In terms of the median of the MSE, the proposed method outperforms if the nowcasting horizon is not greater than 25 minutes. This observation is consistent with the findings obtained from Figure 9 in the previous section. Note that, as the reflectivity growth is extrapolated through an autoregressive model in both space and time, the gain of incorporating reflectivity growth naturally decreases as the nowcasting horizon increases. In addition, since only the pixel arrays with high reflectivity are of interest in practice, we also show the comparison, on the right panel of Figure 10, of the MSE evaluated based on pixel arrays with reflectivity above $35 \mathrm{dBz}$. It is possible to see the advantage of the proposed model for a larger nowcasting horizon, say, 20 minutes or 30 minutes ahead. Since the only major difference between the COTREC method and proposed method is that the
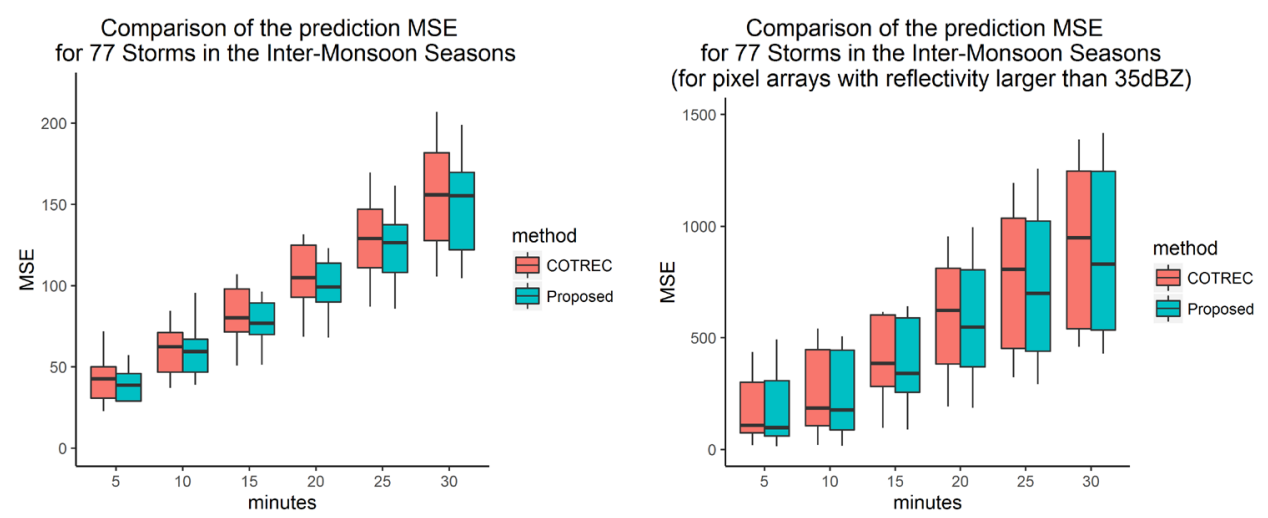

FIG. 10. Box plot of the Mean Squared Error (MSE) of the extrapolated radar reflectivity fields based on the 77 storm events observed from the inter-monsoon season. The horizontal axis indicates the nowcasting horizon ranging from 5 to 30 minutes. In particular, the left panel shows the MSE evaluated based on all pixel arrays in a radar image, while the right panel shows the MSE evaluated based on all pixel arrays with reflectivity larger than $35 \mathrm{dBz}$. 
latter method captures the growth of reflectivity at all pixel arrays, the findings from Figure 10 justifies the need to consider the growth and decay of reflectivity for the space-time extrapolation of weather radar reflectivity field. Also note that, although the COTREC method is used in this paper to obtain the velocity field, the proposed modeling framework allows us to replace COTREC by any other motion estimation methods, such as TITAN, VET and SCIT as reviewed in the Appendix B.

5.2. Southwest monsoon season events. We have different findings based on the data from the Southwest Monsoon Season. The storm events in the Southwest Monsoon Season (June-September) behave in a fundamentally different way as the events in the Inter-Monsoon Season. The scale of thunderstorms within this season is usually bigger and more persistent, meaning that the strength of reflectivity does not change dramatically and the prediction of the motion of weather systems is of the central interest. For example, "Sumatra squalls" are a line of thunderstorms that develop at night over Sumatra, move to the west coast of Peninsula of Malaysia and hit Singapore during the early morning hours. Heavy rain persists for 1-2 hours, followed by cloudy conditions and light rain until afternoon. Hence, the proposed method is not designed for this type of storm events as the modeling of the growth and decay may not be necessary. However, the comparison results are still presented in Figure 11 in order to provide more operational insights. Figure 11 shows the box plot of the Mean Squared Error (MSE) associated with the extrapolated radar reflectivity fields based on the 199 storm events collected from the southwest monsoon season. We see that, for more persistent weather systems, incorporating the growth and decay into the nowcasting
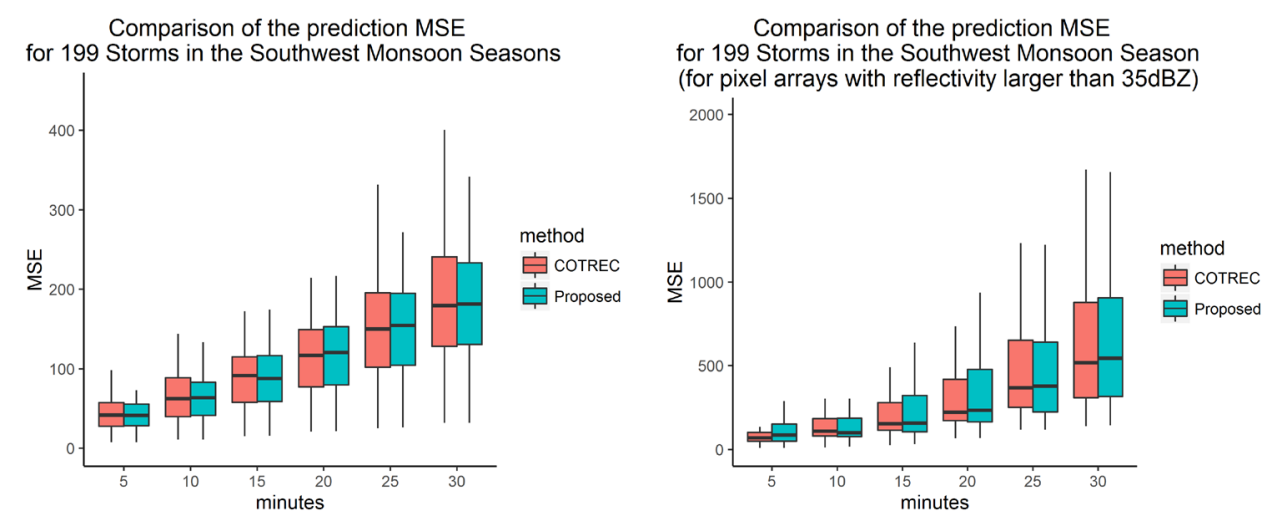

FIG. 11. Box plot of the Mean Squared Error (MSE) of the extrapolated radar reflectivity fields based on the 199 storm events observed from the southwest monsoon season. The horizontal axis indicates the nowcasting horizon ranging from 5 to 30 minutes. In particular, the left panel shows the MSE evaluated based on all pixel arrays in a radar image, while the right panel shows the MSE evaluated based on all pixel arrays with reflectivity larger than $35 \mathrm{dBz}$. 
model does not provide any advantage to the model, and is essentially not necessary.

6. Conclusions and future research. A statistical framework has been proposed for modeling weather radar image data. The two-dimensional radar reflectivity field is modeled by a spatio-temporal space-time conditional autoregressive process. Numerical studies, based on 276 storm events, have been performed to investigate the advantages of the approach and obtain some useful operational insights.

It is worth noting that the main goal of this work is not to develop a new storm tracking algorithm, but to investigate a statistical modeling framework that helps to integrate existing storm tracking algorithms (such as COTREC, TITAN, etc.) and a stochastic spatio-temporal component for reflectivity growth and decay. Hence, it is possible to incorporate other storm tracking algorithms, such TITAN, SCIT, NWP, or even hybrid methods, into the modeling framework. For example, the cell tracking algorithms (such as TITAN) detect discrete objects which consist of contiguous regions of high reflectivity. From each radar image, the locations of the detected objects as well as the associated characteristics are recorded. Hence, for each object, a multivariate time series of its key characteristics are obtained. When we consider multiple objects in the spatio-domain simultaneously, statistical spatio-temporal modeling becomes a powerful tool to exploit the space-time correlation among the objects, that is, thunderstorm cells. Although this is out of the scope of the current paper, it is a good future research topic.

In addition, the paper is only concerned with the modeling of two-dimensional radar reflectivity fields at a fixed altitude (1 km above the sea level), while the vertical motion of weather systems has not been considered. Since a weather system is a dynamic three-dimensional object and modern radar systems are equipped with the capability to return the reflectivity at multiple altitude layers simultaneously, another important future work is to explore the modeling of three-dimensional radar reflectivity fields by extending the proposed method.

\section{APPENDIX A: CONSTRUCTING THE VELOCITY VECTOR FIELD}

The existing pattern-based method for constructing the velocity vector field as well as the translation operators is briefly described. Let a two-dimension discrete random function $Z_{t}\left(k_{1}, k_{2} ; s_{i}\right)$ represent the reflectivity at time $t$ on the $\left(k_{1}, k_{2}\right)$ th pixel within the pixel array centered at $s_{i}$. Here, $k_{1}, k_{2}=1,2, \ldots, 19$ as each pixel arrays consists of $19 \times 19$ pixels. The velocity vector at location $s_{i}$ is given by $s_{j^{*}}-s_{i}$ where $s_{j^{*}}$ is found by maximizing the Pearson's correlation coefficient, $r$, between $Y_{t}\left(\cdot, \cdot ; s_{i}\right)$ and $Y_{t+1}\left(\cdot, \cdot ; s_{j}\right)$, that is,

$$
j^{*}=\underset{j}{\operatorname{argmax}}=r\left(Y_{t}\left(\cdot, \cdot ; s_{i}\right), Y_{t+1}\left(\cdot, \cdot ; s_{j}\right)\right) .
$$


In other words, the Pearson's correlation coefficient between the reflectivity patterns within pixel arrays is computed for all possible pairs of pixel arrays in two successive radar scans respectively taken at times $t$ and $t+1$. Then the pair with the highest correlation is connected forming the velocity vector. One key assumption behind this method is that the shape of the reflectivity image within a pixel array does not change dramatically, which is valid when time interval is short, say, 5 minutes as in this paper.

Let $\tilde{v}_{t}\left(s_{i}\right)=\left(\tilde{v}_{t}^{(1)}\left(s_{i}\right), \tilde{v}_{t}^{(2)}\left(s_{i}\right)\right)^{T}$ be the velocity vector at location $s_{i}$ obtained from equation (A.1), where $\tilde{v}_{t}^{(1)}$ and $\tilde{v}_{t}^{(2)}$ are the horizontal and vertical components of the velocity vector. To improve the consistency of the constructed velocity field, the smoothed velocity vectors are obtained by minimizing the sum of squared error

$$
J_{1}=\int_{S}\left\{\left(\tilde{v}_{t}^{(1)}-v_{t}^{(1)}\right)^{2}+\left(\tilde{v}_{t}^{(2)}-v_{t}^{(2)}\right)^{2}\right\} d x d y
$$

and subject to the Boussineq mass continuity equation

$$
\frac{\partial v_{t}^{(1)}}{\partial x}+\frac{\partial v_{t}^{(2)}}{\partial y}=0
$$

It is known that the constrained minimization problem above is equivalent to the following unconstrained problem [Bertsekas (1982)]:

$$
J_{2}=\int_{S}\left\{\left(\tilde{v}_{t}^{(1)}-v_{t}^{(1)}\right)^{2}+\left(\tilde{v}_{t}^{(2)}-v_{t}^{(2)}\right)^{2}+\lambda\left(\frac{\partial v_{t}^{(1)}}{\partial x}+\frac{\partial v_{t}^{(2)}}{\partial y}\right)\right\} d x d y
$$

which can be efficiently solved using a variational analysis. Here, $\lambda$ is the Lagrangian multiplier.

Once the smoothed velocity vector $v_{t}$ has been found, we formally define the forward and inverse translation operators as follows:

$$
\Gamma \mathbf{x}_{t}=\left(\begin{array}{c}
x_{1, t} \\
x_{2, t} \\
\ldots \\
x_{n, t}
\end{array}\right)+\left(\begin{array}{c}
v_{t}\left(x_{1, t}\right) \\
v_{t}\left(x_{2, t}\right) \\
\ldots \\
v_{t}\left(x_{n, t}\right)
\end{array}\right)=\mathbf{x}_{t+1}
$$

and

$$
\Gamma^{-1} \mathbf{x}_{t+1}=\left(\begin{array}{c}
x_{1, t+1} \\
x_{2, t+1} \\
\ldots \\
x_{n, t+1}
\end{array}\right)-\left(\begin{array}{c}
v_{t}\left(x_{1, t}\right) \\
v_{t}\left(x_{2, t}\right) \\
\ldots \\
v_{t}\left(x_{n, t}\right)
\end{array}\right)=\mathbf{x}_{t} .
$$




\section{APPENDIX B: A SUMMARY OF EXISTING STORM TRACKING ALGORITHMS}

We provide a brief summary of the existing storm tracking algorithms. Since a large number of methods have been proposed in the past few decades, it is impossible to include all methods in this survey. Hence, we only include those widely used ones based on the following four categories:

- Lagrangian persistence (the most fundamental method, but does not provide good results).

- AREA tracking, including the following algorithms:

- TREC (Tracking Radar Echoes by Correlation) and its variations such as COTREC. This is the method adopted in this paper.

- VET (Variational Echo Tracking).

- CELL tracking, including the following algorithms:

- TITAN (Thunderstorm Identification Tracking Analysis and Nowcasting) and its variations.

- SCIT (Storm Cell Identification and Tracking).

- TRT (Thunderstorm Radar Tracking).

- Other methods such as TRACED3D and CELLTRACK.

- Other categories including the Spectral methods, expert systems, etc.

Table 3 summarizes the major operational QPF systems in different countries. It is seen that, cell tracking and area tracking are the most widely used strategies for estimating the motion of a storm system. Each strategy has its own advantages when they are applied to a certain type of weather system and for certain purposes.

TABLE 3

A summary of some major operational systems for quantitative precipitation forecast in different countries

\begin{tabular}{|c|c|c|c|c|}
\hline System & Country & Year & Method & Category \\
\hline AMV & Finland & 2000 & TREC & AREA tracking \\
\hline ANC & United States & 2003 & TREC + TITAN & $\begin{array}{l}\text { AREA and CELL } \\
\text { tracking (hybrid) }\end{array}$ \\
\hline CARDS & Canada & & TITAN & CELL tracking \\
\hline Czech system & Czech & 2007 & COTREC + CELLTRACK & $\begin{array}{l}\text { AREA and CELL } \\
\text { tracking (hybrid) }\end{array}$ \\
\hline GANDOLF & U.K. & 2004 & Optical Flow (OF) & Lagrangian persistence \\
\hline MAPLE & Canada & 2004 & VET & AREA tracking \\
\hline NIMROD & U.K. & 1998 & track CRAs & AREA tracking \\
\hline S-PROG & Austria/Spain & 2003 & & Spectral approach \\
\hline STEPS & Austria/U.K. & 2006 & Optical Flow & Lagrangian persistence \\
\hline SWIRLS & Hong Kong & 2004 & COTREC & AREA tracking \\
\hline TRT & Switzerland & 2004 & TRT & CELL tracking \\
\hline
\end{tabular}




\section{APPENDIX C: AN ILLUSTRATION OF CONVERTING RADAR REFLECTIVITY TO RAIN RATE}

The Z-R relationships relate the value of the measured reflectivity to the value of the rain rate according to the following general formula by Marshall and Palmer [Marshall and Palmer (1948)],

$$
R=\left(\frac{10^{\frac{Z}{10}}}{200}\right)^{\frac{5}{8}},
$$

where $Z$ is the radar reflectivity in $\mathrm{dBZ}$ and $R$ is the rain rate given by $\mathrm{mm} / \mathrm{hr}$.

To illustrate the conversion of radar reflectivity to rain rate, Figure 12 shows the rain rate converted from the predicted reflectivity using the proposed method shown in Figure 8. It should be noted that the Marshall and Palmer relationship is just an empirical relationship and dramatic changes in parameters are often needed within an individual storm as well as between storms of different types. Different weather service agencies in the world typically use their own modified radarrainfall relationship. The calibration of the radar-rainfall relationship is not of the interest of this paper, and readers may refer to Brown et al. (2001) and Fuentes, Reich and Lee (2008) for the space-time calibration of radar-rainfall data using advanced statistical methods.

\section{APPENDIX D: CODE}

The source code for the proposed spatio-temporal approach described in the paper is available on the public repositories of GitHub (https://github.com/
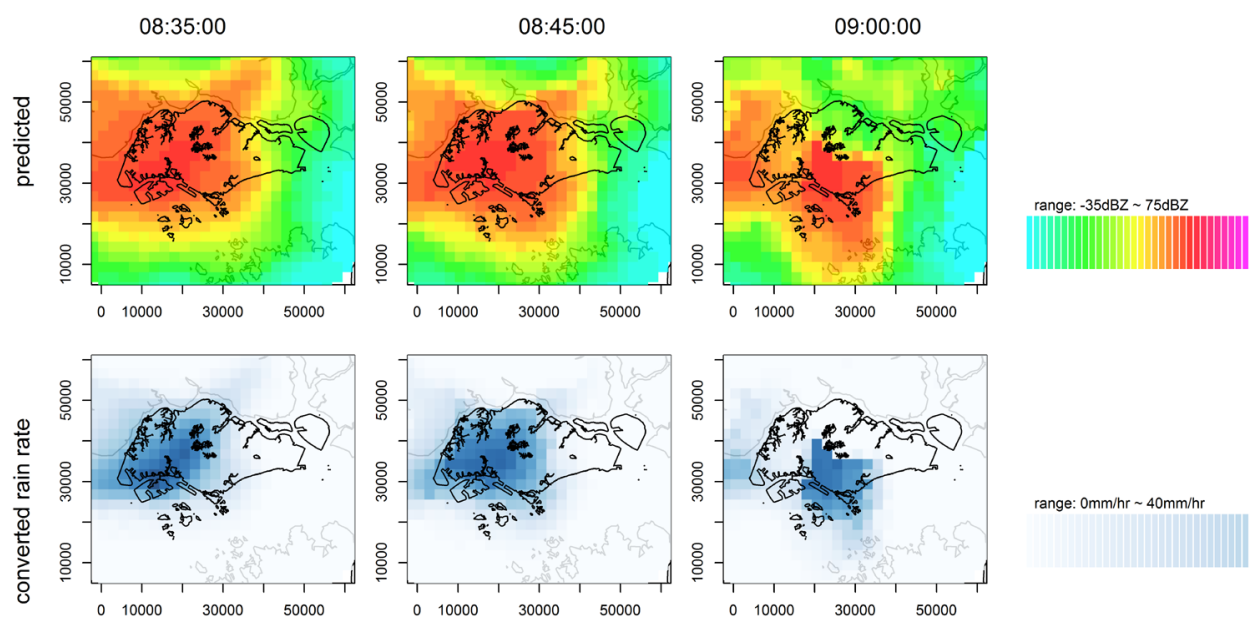

FIG. 12. Converted rain rate $(\mathrm{mm} / \mathrm{hr})$ at 8:35 AM, 8:45 AM, and 9:00 AM. The first row shows the predicted reflectivity using the proposed approach, while the second row shows the rain rate converted from the predicted reflectivity using the Marshall-Palmer relationship. 
liuxiaodnn/GitHub.git). The $\mathrm{R}$ code can be browsed and downloaded by users. A testing dataset is also provided in order to run the code.

Acknowledgements. The authors would like to thank the National Environment Agency of Singapore for supporting the research reported in this paper. The authors would also like to thank the Editor, Associate Editor, and two referees for their constructive comments that greatly helped to improve the quality of the paper.

\section{REFERENCES}

AsSUnÇãO, R. and KRAINSKI, E. (2009). Neighborhood dependence in Bayesian spatial models. Biom. J. 51 851-869. MR2751717

BanerJee, S., Carlin, B. P. and Gelfand, A. E. (2015). Hierarchical Modeling and Analysis for Spatial Data, 2nd ed. Monographs on Statistics and Applied Probability 135. CRC Press, Boca Raton, FL. MR3362184

BERTSEKAS, D. P. (1982). Constrained Optimization and Lagrange Multiplier Methods. Academic Press, Boston. MR0690767

BESAG, J. and KOOPERBERG, C. (1995). On conditional and intrinsic autoregressions. Biometrika 82 733-746. MR1380811

Bowler, N. E. H., PIERCE, C. E. and SEED, A. (2004). Development of a precipitation nowcasting algorithm based upon optical flow techniques. J. Hydrol. 288 74-91.

Bowler, N. E., Pierce, C. E. and Seed, A. W. (2006). A probabilistic precipitation forecasting scheme which merges an extrapolation nowcast with downscaled NWP. Q. J. R. Meteorol. Soc. 132 2127-2155.

Brown, P. E., Diggle, P. J., Lord, M. E. and Young, P. C. (2001). Space-time calibration of radar rainfall data. J. Roy. Statist. Soc. Ser. C 50 221-241. MR1833274

Browning, K. A., Collier, C. G., Lark, P. R., Menmuir, P., Monk, G. A. and OwENS, R. G. (1982). On the forecasting of frontal rain using a weather radar network. Mon. Weather Rev. 110 534-552.

CARlin, B. P. and BANerJeE, S. (2003). Hierarchical multivariate CAR models for spatiotemporally correlated survival data (with discussion). In Bayesian Statistics, 7 (Tenerife, 2002) 45-63. Oxford Univ. Press, New York. MR2003166

Cressie, N. A. C. (1993). Statistics for Spatial Data. Wiley, New York. MR1239641

DiXON, M. and WIENER, G. (1993). TITAN: Thunderstorm identification, traking, analysis, and nowcasting-a radar-based methodology. J. Atmos. Ocean. Technol. 10 785-797.

Fuentes, M., Reich, B. and LEE, G. (2008). Spatial-temporal mesoscale modeling of rainfall intensity using gage and radar data. Ann. Appl. Stat. 2 1148-1169. MR2655653

GelPKE, V. and KÜNSCH, H. R. (2001). Estimation of motion from sequences of images. In Spatial Statistics: Methodological Aspects and Applications. Lect. Notes Stat. 159 141-167. Springer, New York. MR1847746

GERMANN, U. and ZAWADZKI, I. (2002). Scale-dependence of the predicability of precipitation from continental radar images. Part I: Description of the methodology. Mon. Weather Rev. 130 $2859-2873$.

Haining, R. (1990). Spatial Data Analysis in the Social and Environmental Sciences. Cambridge Univ. Press, Cambridge.

HAN, L., Fu, S. X., ZhaO, L. F., Zheng, Y. G., WANG, H. Q. and LiN, Y. J. (2009). 3D convective storm identification, tracking, and forecasting-an enhanced TITAN algorithm. J. Atmos. Ocean. Technol. 26 719-732. 
Hartigan, J. A. and Wong, M. A. (1979). A K-means clustering algorithm. Appl. Stat. 28 100108.

HoRn, B. K. P. and SCHUncK, B. G. (1981). Determining optical flow. Artificial Intelligence 17 185-203.

Jin, X., CARLIN, B. P. and BANERJEE, S. (2005). Generalized hierarchical multivariate CAR models for areal data. Biometrics 61 950-961. MR2216188

LeESE, J. A., NovAK, C. S. and ClARK, B. B. (1971). An automated technique for obtaining cloud motion from geosynchronous saellite data using cross correlation. J. Appl. Meteorol. 10 118-132.

LI, P. and LAI, S. T. (2004). Short-range quantitative precipitation forecasting in Hong Kong. J. Hydrol. 288 189-209.

LI, L., SCHMID, W. and Joss, J. (1995). Nowcasting of motion and growth of precipitation with radar over a complex orography. J. Appl. Meteorol. 34 1286-1300.

MARIELla, L. and TARANTINO, M. (2010). Spatial temporal conditional auto-regressive model: A new autoregressive matrix. Aust. J. Stat. 39 223-244.

Marshall, J. S. and PALmer, W. M. (1948). The distribution of raindrops with size. J. Meteorol. 5 165-166.

National Environmental Agency Singapore (2014). Weather Statistics. Available at http: //app2.nea.gov.sg/weather-climate/climate-information/weather-statistics.

National EnVironmental Agency Singapore (2017). Challenges in Weather Forecasting. Available at http://www.nea.gov.sg/training-knowledge/weather-climate/weather-forecast/ challenges-in-weather-forecasting/.

RAdHAKRIShnA, B., ZAWAdZKi, I. and FABry, F. (2012). Predictability of precipitation from continental rada images. Part V: Growth and decay. J. Atmos. Sci. $693336-3349$.

RineharT, R. E. and GARVEY, E. T. (1978). Three-dimensional storm motion detection by conventional weather radar. Nature $273287-289$.

Royal Meteorological Institute of Belgium (2008). Quantitative Precipitation Forecasts based on radar observations: Principles. algorithms and operational systems, 2008/0224/52.

SEED, A. W. (2003). A dynamic and spatial scaling approach to advection forecasting. J. Appl. Meteorol. 42 381-388.

Seed, A. W., Pierce, C. E. and Norman, K. (2013). Formulation and evaluation of a scale decomposition-based stochastic precipitation nowcast scheme. Water Resour. Res. 49 6624-6641.

SigRIST, F., KÜNSCH, H. R. and STAHEL, W. A. (2012). A dynamic nonstationary spatio-temporal model for short term prediction of precipitation. Ann. Appl. Stat. 6 1452-1477. MR3058671

StAniforth, A. and Cote, J. (1991). Semi-Lagrangian integration schemes for atmospheric models-a review. Mon. Weather Rev. 119 2206-2223.

STERN, H. and CRESSIE, N. (2000). Posterior predictive model checks for disease mapping models. Stat. Med. 19 2377-2397.

Stroud, J. R., MÜller, P. and SAnsó, B. (2001). Dynamic models for spatiotemporal data. J. R. Stat. Soc. Ser. B. Stat. Methodol. 63 673-689. MR1872059

Testik, F. and Gebremichael, M. (2013). Rainfall: State of the Science. American Geophysical, Union.

WALL, M. M. (2004). A close look at the spatial structure implied by the CAR and SAR models. J. Statist. Plann. Inference 121 311-324. MR2038824

Wilson, J. W., Crook, N. A., Mueller, C. K., Sun, J. and Dixon, M. (1998). Nowcasting thunderstorms: A status report. Bull. Am. Meteorol. Soc. 78 2079-2099.

Wolfson, M. M., Forman, B. E., Hallowell, R. G. and Moore, M. P. (1999). The Growth and Decay Storm Tracker, The 8th Conference on Aviation, Range, and Aerospace Meteorology.

XU, K., WiKLE, C. K. and FoX, N. I. (2005). A kernel-based spatio-temporal dynamical model for nowcasting weather radar reflectivities. J. Amer. Statist. Assoc. 100 1133-1144. MR2236929 
X. LIU

DEPARTMENT OF INDUSTRIAL ENGINEERING

4207 BELL ENGINEERING CENTER

UNIVERSITY OF ARKANSAS

FAYETTEVILle, ARKansas 72701

USA

E-MAIL: liuxiao314923@gmail.com
V. GOPAL

DEPARTMENT OF STATISTICS

AND APPLIEd PRoBABILITY

Block S 16, LeVel 7, 6 SCIENCE Drive 2

NATIONAL UNIVERSITY OF SINGAPORE

SINGAPORE 117546

SINGAPORE

E-MAIL: stavg@nus.edu.sg

J. KALAGNANAM

IBM THOMAS J. WATSON RESEARCH CENTER

1101 KiTCHAWAN RoAD

YORKTOWN HEIGHTS, NEW YORK 10598

USA

E-MAIL: jayant@us.ibm.com 